\title{
Excited heavy meson decays to light vector mesons: Implications for spectroscopy
}

\author{
S. Campanella, P. Colangelo, and F. De Fazio \\ Istituto Nazionale di Fisica Nucleare, Sezione di Bari, Via Orabona 4, I-70126 Bari, Italy
}

(Received 15 October 2018; published 26 December 2018)

\begin{abstract}
We analyze strong decays of excited charmed and beauty mesons into a light vector meson, exploiting the effective field theory based on heavy quark (HQ) symmetries for heavy mesons, and on the hidden gauge symmetry to incorporate light vector mesons. HQ symmetries allow us to classify the heavy mesons in spin doublets, and to relate decays of excited states. We build effective Lagrangian terms governing the $\mathcal{H}_{i} \rightarrow P^{(*)} V$ modes, with $\mathcal{H}_{i}$ an excited $s, p, d$, and $f$-wave heavy-light quark meson, $P, P^{*}$ the lowest-lying $J^{P}=\left(0^{-}, 1^{-}\right)$heavy-light mesons, and $\mathrm{V}$ a light vector meson. Predictions are provided for ratios of decay widths that are independent of the strong couplings in the effective Lagrangian terms. A classification of the newly observed heavy-light mesons is proposed.
\end{abstract}

DOI: 10.1103/PhysRevD.98.114028

\section{INTRODUCTION}

Great progress has been achieved in heavy hadron spectroscopy, thanks to the efforts of several experimental groups at different facilities which have provided new pieces of information [1]. In the open charm meson spectrum the two lowest-lying (1S) and the four 1P orbital excitations are identified, both for nonstrange and strange mesons [2]. Information is available for larger mass states which could be identified either with higher orbital or radial excitations. Experimental observations are less abundant in the case of beauty mesons: the established states are the two lowest-lying (1S) states and two among the four 1P orbital excitations, both with and without strangeness [2]. There is progress also in baryon spectroscopy, with the observation of five new narrow $\Omega_{c}$ resonances [3] and of the doubly charmed $\Xi_{c c}$ [4]: however, in this paper we are only concerned with mesons.

Prompt production and production in $B$ decays, the main production mechanisms of excited charmed mesons, provide us with different and complementary information. Prompt production allows us to establish if a state has natural $\left(J^{P}=0^{+}, 1^{-}, 2^{+}, \ldots\right)$ or unnatural $\left(J^{P}=0^{-}, 1^{+}, 2^{-}, \ldots\right)$ parity, while spin-parity can be determined by Dalitz plot analyses in $B$ decay production. On the other hand, it is possible to measure ratios of branching fractions of strong

Published by the American Physical Society under the terms of the Creative Commons Attribution 4.0 International license. Further distribution of this work must maintain attribution to the author(s) and the published article's title, journal citation, and DOI. Funded by SCOAP ${ }^{3}$. decay modes, information that can be used to classify the decaying meson, as we are going to discuss.

Several observed open charm mesons are awaiting for a proper identification. In Table I we include the resonances observed by the BABAR Collaboration (in 2010) in the inclusive production of $D^{+} \pi^{-}, D^{0} \pi^{+}$and $D^{*+} \pi^{-}$[5]. The $\mathrm{LHCb}$ Collaboration performed a similar analysis (in 2013), with the findings in Table II [6]. LHCb also carried out (in 2016) a Dalitz plot analysis of $B^{-} \rightarrow D^{+} \pi^{-} \pi^{-}$, reporting evidence of the resonances in Table III [7]. Many of the states found in the different analyses are likely to be the same, namely the BABAR $D^{0}(2550)$ and $D^{* 0}(2600)$ states in Table I coincide with the LHCb ones $D_{J}^{0}(2580)$ and $D_{J}^{* 0}(2650)$ in Table II. $D_{1}^{* 0}(2680)$ in Table III is probably different from $D^{* 0}(2600)$, although presumably they both have $J^{P}=1^{-}$. The identification of $D^{0}(2750)$ in Table I with $D_{J}^{0}(2740)$ in Table II is also plausible. Two different resonances are present in the mass range around $2760 \mathrm{MeV}$ : one having $J^{P}=1^{-}$and another one with $J^{P}=3^{-}$. The state in Table III is definitely the latter one, reported by $\mathrm{LHCb}$ [8]. In the cases of mesons with mass

TABLE I. Mass, width, and spin parity of charmed resonances observed by the BABAR Collaboration [5].

\begin{tabular}{lccc}
\hline \hline Resonance & Mass $(\mathrm{MeV})$ & $\Gamma(\mathrm{MeV})$ & $J^{P}$ \\
\hline$D^{0}(2550)$ & $2539.4 \pm 4.5 \pm 6.8$ & $130 \pm 12 \pm 13$ & $0^{-}$ \\
$D^{* 0}(2600)$ & $2608.7 \pm 2.4 \pm 2.5$ & $93 \pm 6 \pm 13$ & Natural \\
$D^{*+}(2600)$ & $2621.3 \pm 3.7 \pm 4.2$ & 93 (fixed) & Natural \\
$D^{0}(2750)$ & $2752.4 \pm 1.7 \pm 2.7$ & $71 \pm 6 \pm 11$ & \\
$D^{* 0}(2760)$ & $2763.3 \pm 2.3 \pm 2.3$ & $60.9 \pm 5.1 \pm 3.6$ & Natural \\
$D^{*+}(2760)$ & $2769.7 \pm 3.8 \pm 1.5$ & 60.9 (fixed) & Natural \\
\hline \hline
\end{tabular}


TABLE II. Mass, width, and spin parity of charmed mesons from the LHCb analysis of inclusive $D^{(*)} \pi$ production [6].

\begin{tabular}{lccc}
\hline \hline Resonance & Mass $(\mathrm{MeV})$ & $\Gamma(\mathrm{MeV})$ & $J^{P}$ \\
\hline$D_{J}^{0}(2580)$ & $2579.5 \pm 3.4 \pm 5.5$ & $177.5 \pm 17.7 \pm 46.0$ & Unnatural \\
$D_{J}^{* 0}(2650)$ & $2649.2 \pm 3.5 \pm 3.5$ & $140.2 \pm 17.1 \pm 18.6$ & Natural \\
$D_{J}^{0}(2740)$ & $2737.0 \pm 3.5 \pm 11.2$ & $73.2 \pm 13.4 \pm 25.0$ & Unnatural \\
$D_{J}^{* 0}(2760)$ & $2761.1 \pm 5.1 \pm 6.5$ & $74.4 \pm 4.3 \pm 37.0$ & Natural \\
$D_{J}^{* 0}(2760)$ & $2760.1 \pm 1.1 \pm 3.7$ & $74.4 \pm 3.4 \pm 19.1$ & Natural \\
$D_{J}^{*+}(2760)$ & $2771.7 \pm 1.7 \pm 3.8$ & $66.7 \pm 6.6 \pm 10.5$ & Natural \\
$D_{J}^{0}(3000)$ & $2971.8 \pm 8.7$ & $188.1 \pm 44.8$ & Unnatural \\
$D_{J}^{* 0}(3000)$ & $3008.1 \pm 4.0$ & $110.5 \pm 11.5$ & Natural \\
$D_{J}^{*+}(3000)$ & $3008.1($ fixed $)$ & $110.5($ fixed $)$ & Natural \\
\hline \hline
\end{tabular}

TABLE III. Mass, width, and spin parity of charmed mesons observed by LHCb in Dalitz plot analysis of $B^{-} \rightarrow D^{+} \pi^{-} \pi^{-}$[7].

\begin{tabular}{lccc}
\hline \hline Resonance & Mass $(\mathrm{MeV})$ & $\Gamma(\mathrm{MeV})$ & $J^{P}$ \\
\hline$D_{1}^{* 0}(2680)$ & $2681.1 \pm 5.6 \pm 4.9 \pm 13.1$ & $186.7 \pm 8.5 \pm 8.6 \pm 8.2$ & $1^{-}$ \\
$D_{3}^{* 0}(2760)$ & $2775.5 \pm 4.5 \pm 4.5 \pm 4.7$ & $95.3 \pm 9.6 \pm 7.9 \pm 33.1$ & $3^{-}$ \\
$D_{2}^{* 0}(3000)$ & $3214 \pm 29 \pm 33 \pm 36$ & $186 \pm 39 \pm 34 \pm 63$ & $2^{+}$ \\
\hline \hline
\end{tabular}

close to $3000 \mathrm{MeV}$ LHCb has not provided a systematic uncertainty for the parameters in Table II, since the states are observed at the limit of the considered mass range. The latest results for strange charmed mesons are in Table IV.

While spin parity of charmed mesons can be established by the amplitude analyses in production in $B$ decays, for beauty mesons the quantum number assignment is more difficult. In addition to the above-mentioned established states, recent observations are due to $\mathrm{CDF}$ and $\mathrm{LHCb}$ Collaborations. CDF found a state named $B(5970)$ [13], likely the same as $B_{J}^{0,+}(5960)$ observed by LHCb together with $B_{J}^{0,+}(5840)$ decaying to $B^{+} \pi^{-}, B^{0} \pi^{+}$[14]. Spin parity is not established, and mass and width are affected by large uncertainties: the values from PDG fits [2] are in Table V. The identification as $2 \mathrm{~S}$ excitations has been proposed [14]. New results on $B_{s 1}(5830)$ and $B_{s 2}^{*}(5840)$ have also been obtained by the CMS Collaboration [15].

In [16] a comprehensive analysis of the open charm and open beauty mesons was performed based on the classification scheme in the heavy quark limit, attempting to fit the observed states in this scheme. Information on the

TABLE IV. Mass, width, and spin parity of the latest observed strange charmed mesons.

\begin{tabular}{lcccc}
\hline \hline Resonance & Mass $(\mathrm{MeV})$ & $\Gamma(\mathrm{MeV})$ & $J^{P}$ & Ref. \\
\hline$D_{s 1}^{*}(2700)$ & $2709.2 \pm 1.9 \pm 4.5$ & $115.8 \pm 7.3 \pm 12.1$ & $1^{-}$ & {$[9]$} \\
$D_{s 1}^{*}(2700)$ & $2699 \pm \pm_{7}^{14}$ & $127 \pm \pm_{19}^{24}$ & $1^{-}$ & {$[10]$} \\
$D_{s 1}^{*}(2860)$ & $2859 \pm 12 \pm 6 \pm 23$ & $159 \pm 23 \pm 27 \pm 72$ & $1^{-}$ & {$[11]$} \\
$D_{s 3}^{*}(2860)$ & $2860.5 \pm 2.6 \pm 2.5 \pm 6$ & $53 \pm 7 \pm 4 \pm 6$ & $3^{-}$ & {$[11]$} \\
$D_{s J}(3040)$ & $3044 \pm 8 \pm_{5}^{30}$ & $239 \pm 35 \pm_{42}^{46}$ & & {$[12]$} \\
\hline \hline
\end{tabular}

TABLE V. PDG fit for the mass and width of nonstrange beauty mesons with uncertain classification [2].

\begin{tabular}{lcl}
\hline \hline Resonance & Mass $(\mathrm{MeV})$ & $\Gamma(\mathrm{MeV})$ \\
\hline$B_{J}^{+}(5840)$ & $5851 \pm 19$ & $571 \pm 19$ \\
$B_{J}^{0}(5840)$ & $5863 \pm 9$ & $584 \pm 9$ \\
$B_{J}^{+}(5970)$ & $5964 \pm 5$ & $685 \pm 5$ \\
$B_{J}^{0}(5970)$ & $5971 \pm 5$ & $691 \pm 5$ \\
\hline \hline
\end{tabular}

strong decay modes to $D_{(s)} M$ or $D_{(s)}^{*} M$, with $M$ a light pseudoscalar meson, was exploited, and the states in Table I and most of those in Table IV were considered. In the same approach, studies for the states in Tables II and III observed after the analysis in [16] have been carried out in $[17,18]$.

More data are still needed for classification, which is a nontrivial task for the newly observed mesons. If the resonance mass is large enough, several decay channels are open, in particular those with a light final vector meson which provide an important new piece of information. This paper is devoted to such a phenomenology.

In the next section we restate the theoretical framework based on heavy quark (HQ) symmetries to describe spectrum and decay processes. For transitions into light pseudoscalars, effective Lagrangians are written exploiting the HQ symmetries and the (spontaneoulsy broken) chiral symmetry holding in QCD for massless $u, d, s$ quarks, with the light pseudoscalar mesons being the pseudo-Goldstone bosons. The approach can be extended to incorporate the light vector mesons, treated as gauge fields of a hidden local symmetry. In Sec. III we construct the effective Lagrangians describing strong heavy-light meson decays with the emission of a light vector meson, generalizing the analysis in $[19,20]$. We give the expressions for $\mathcal{H}_{i} \rightarrow$ $P^{(*)} V$ decay rates, with $\mathrm{V}$ a light vector meson, $P^{(*)}$ the lowest-lying $J^{P}=\left(0^{-}, 1^{-}\right)$heavy-light mesons, and $\mathcal{H}_{i}$ either a orbital or a radial heavy-light excitation. Sections IV and V contain numerical analyses for charmed and beauty mesons, considering states requiring identification and making predictions for heavier excitations. Relations among decay rates, independent of the hadronic couplings, are constructed: they are suitable for experimental measurements and for classifications. The conclusions are presented in the last section.

\section{THEORETICAL SETUP}

\section{A. Heavy-light meson decays to light pseudoscalar mesons}

The physics of hadrons containing a single heavy quark can be systematically analyzed considering the $m_{Q} \rightarrow \infty$ heavy quark (HQ) mass limit, formalized in the heavy quark effective theory (HQET) [21]. In such a limit, two symmetries emerging in QCD can be exploited: the heavy quark spin symmetry, allowing one to relate the properties 
of hadrons which only differ for the heavy quark spin orientation, and the heavy quark flavor symmetry, relating the properties of hadrons which only differ for the heavy quark flavor. The classification of heavy-light $Q \bar{q}$ mesons ( $\bar{q}$ a light antiquark) in the HQ limit is based on the decoupling of the heavy quark spin $s_{Q}$ from the total angular momentum $s_{\ell}$ of the light degrees of freedom (light quarks and gluons). Since such angular momenta are separately conserved in strong interaction processes, the heavy mesons can be classified in doublets of different $s_{\ell}$. Each doublet comprises two states, spin partners, with total spin $J=s_{\ell} \pm \frac{1}{2}$ and parity $P=(-1)^{\ell+1}, \ell$ being the orbital angular momentum of the light degrees of freedom and $\vec{s}_{\ell}=\vec{\ell}+\vec{s}_{q}\left(s_{q}\right.$ the light antiquark spin). In the HQ limit the spin partners in each doublet are degenerate and, due to flavor symmetry, the properties of the states in a doublet are related to those of the corresponding states differing for the heavy flavor.

Meson doublets corresponding to $\ell=0,1,2$, and 3 are those referred to as $s, p, d$, and $f$ wave states in the constituent quark model. The lowest lying $Q \bar{q}$ mesons correspond to $\ell=0$, hence $s_{\ell}^{P}=\frac{1}{2}^{-}$; the doublet consists of two states with $J^{P}=\left(0^{-}, 1^{-}\right)$, denoted as $\left(P, P^{*}\right)$. For $\ell=1$ one has $s_{\ell}^{P}=\frac{1}{2}^{+}$and $s_{\ell}^{P}=\frac{3}{2}^{+}$. The two doublets have $J^{P}=\left(0^{+}, 1^{+}\right)$and $J^{P}=\left(1^{+}, 2^{+}\right)$, respectively; the members of the $J_{s_{\ell}}^{P}=\left(0^{+}, 1^{+}\right)_{1 / 2}$ doublet are denoted as $\left(P_{0}^{*}\right.$, $\left.P_{1}^{\prime}\right)$, those of the $J_{s_{\ell}}^{P}=\left(1^{+}, 2^{+}\right)_{3 / 2}$ doublet as $\left(P_{1}, P_{2}^{*}\right)$. For $\ell=2$ one has $s_{\ell}^{P}=\frac{3}{2}^{-}$, and $s_{\ell}^{P}=\frac{5}{2}^{-}$; the first doublet comprises $\left(P_{1}^{*}, P_{2}\right)$ with $J^{P}=\left(1^{-}, 2^{-}\right)$, the second one the states $\left(P_{2}^{\prime}, P_{3}^{*}\right)$ with $J^{P}=\left(2^{-}, 3^{-}\right)$. One can continue with $\ell=3$, which gives $s_{\ell}^{P}=\frac{5}{2}^{+}$and $s_{\ell}^{P}=\frac{7}{2}^{+}$: here we only consider the first one of such doublets, which comprises two states with $J^{P}=\left(2^{+}, 3^{+}\right)$, denoted as $\left(P_{2}^{*}, P_{3}\right)$. The same classification holds for radial excitations: for them we use the same notation, but for a tilde $\left(\tilde{P}, \tilde{P}^{*}, \ldots\right)$.

Effective Lagrangians describing the strong interactions of such mesons can be constructed introducing effective fields for each doublet, following e.g., the procedure based on the covariant representation of the states [22]. We denote by $H_{a}\left(a=u, d, s\right.$ a light flavor index) the $s_{\ell}^{P}=\frac{1}{2}^{-}$doublet, $S_{a}$ and $T_{a}$ the $s_{\ell}^{P}=\frac{1}{2}{ }^{+}$and $s_{\ell}^{P}=\frac{3}{2}{ }^{+}$doublets, $X_{a}$ the $s_{\ell}^{P}=\frac{3}{2}^{-}, X_{a}^{\prime}$ the $s_{\ell}^{P}=\frac{5}{2}^{-}$and $F_{a}$ the $s_{\ell}^{P}=\frac{5}{2}^{+}$doublets. The corresponding effective fields read

$$
\begin{aligned}
H_{a} & =\frac{1+\mathrm{y}}{2}\left[P_{a \mu}^{*} \gamma^{\mu}-P_{a} \gamma_{5}\right], \\
S_{a} & =\frac{1+\mathrm{y}}{2}\left[P_{1 a}^{\prime \mu} \gamma_{\mu} \gamma_{5}-P_{0 a}^{*}\right], \\
T_{a}^{\mu} & =\frac{1+\mathrm{y}}{2}\left\{P_{2 a}^{\mu \nu} \gamma_{\nu}-P_{1 a \nu} \sqrt{\frac{3}{2}} \gamma_{5}\left[g^{\mu \nu}-\frac{1}{3} \gamma^{\nu}\left(\gamma^{\mu}-v^{\mu}\right)\right]\right\}, \\
X_{a}^{\mu} & =\frac{1+\mathrm{y}}{2}\left\{P_{2 a}^{* \mu \nu} \gamma_{5} \gamma_{\nu}-P_{1 a \nu}^{\prime *} \sqrt{\frac{3}{2}}\left[g^{\mu \nu}-\frac{1}{3} \gamma^{\nu}\left(\gamma^{\mu}+v^{\mu}\right)\right]\right\}, \\
X_{a}^{\prime \mu \nu} & =\frac{1+\mathrm{y}}{2}\left\{P_{3 a}^{* \mu \nu \sigma} \gamma_{\sigma}-P_{2 a}^{\prime \alpha \beta} \sqrt{\frac{5}{3}} \gamma_{5}\left[g_{\alpha}^{\mu} g_{\beta}^{\nu}-\frac{1}{5} \gamma_{\alpha} g_{\beta}^{\nu}\left(\gamma^{\mu}-v^{\mu}\right)-\frac{1}{5} \gamma_{\beta} g_{\alpha}^{\mu}\left(\gamma^{\nu}-v^{\nu}\right)\right]\right\}, \\
F_{a}^{\mu \nu} & =\frac{1+\mathrm{y}}{2}\left\{P_{3 a}^{\mu \nu \sigma} \gamma_{\sigma} \gamma_{5}-P_{2 a}^{\prime * \alpha \beta} \sqrt{\frac{5}{3}}\left[g_{\alpha}^{\mu} g_{\beta}^{\nu}-\frac{1}{5} \gamma_{\alpha} g_{\beta}^{\nu}\left(\gamma^{\mu}-v^{\mu}\right)-\frac{1}{5} \gamma_{\beta} g_{\alpha}^{\mu}\left(\gamma^{\nu}-v^{\nu}\right)\right]\right\} .
\end{aligned}
$$

$v$ is the meson four-velocity, conserved in strong interactions. The operators in Eq. (1) contain a factor $\sqrt{m_{Q}}$, have dimension $3 / 2$ and annihilate mesons with four-velocity $v$.

The octet of light pseudoscalar mesons is introduced defining $\xi=e^{\frac{i \mathcal{M}}{f_{\pi}}}$ and $\Sigma=\xi^{2}$, with the matrix $\mathcal{M}$ comprising $\pi, K$, and $\eta$ fields $\left(f_{\pi}=132 \mathrm{MeV}\right)$ :

$$
\mathcal{M}=\left(\begin{array}{ccc}
\sqrt{\frac{1}{2}} \pi^{0}+\sqrt{\frac{1}{6} \eta} & \pi^{+} & K^{+} \\
\pi^{-} & -\sqrt{\frac{1}{2}} \pi^{0}+\sqrt{\frac{1}{6}} \eta & K^{0} \\
K^{-} & \bar{K}^{0} & -\sqrt{\frac{2}{3} \eta}
\end{array}\right) .
$$

Vector and axial-vector currents can be defined as

$$
\begin{aligned}
& \mathcal{V}_{\mu b a}=\frac{1}{2}\left(\xi^{\dagger} \partial_{\mu} \xi+\xi \partial_{\mu} \xi^{\dagger}\right)_{b a}, \\
& \mathcal{A}_{\mu b a}=\frac{i}{2}\left(\xi^{\dagger} \partial_{\mu} \xi-\xi \partial_{\mu} \xi^{\dagger}\right)_{b a},
\end{aligned}
$$

and under the chiral group $S U(3)_{L} \times S U(3)_{R}$ the transformation properties are

$$
\begin{gathered}
\xi \rightarrow L \xi U^{\dagger}=U \xi R^{\dagger}, \\
\mathcal{A}_{\mu} \rightarrow U \mathcal{A}_{\mu} U^{\dagger},
\end{gathered}
$$




$$
\mathcal{V}_{\mu} \rightarrow U \mathcal{V}_{\mu} U^{\dagger}+U \partial_{\mu} U^{\dagger}
$$

With the definition

$$
D_{\mu b a}=-\delta_{b a} \partial_{\mu}+\mathcal{V}_{\mu b a},
$$

at the leading order in the heavy quark mass expansion and in the light meson momentum, the effective Lagrangian terms invariant under heavy quark spin flavor and light quark chiral transformations can be constructed [23-27]:

$$
\begin{gathered}
\mathcal{L}_{H}=g \operatorname{Tr}\left[\bar{H}_{a} H_{b} \gamma_{\mu} \gamma_{5} \mathcal{A}_{b a}^{\mu}\right], \\
\mathcal{L}_{S}=h \operatorname{Tr}\left[\bar{H}_{a} S_{b} \gamma_{\mu} \gamma_{5} \mathcal{A}_{b a}^{\mu}\right]+\text { H.c., } \\
\mathcal{L}_{T}=\frac{h^{\prime}}{\Lambda_{\chi}} \operatorname{Tr}\left[\bar{H}_{a} T_{b}^{\mu}\left(i D_{\mu} \mathcal{A}+i D \mathcal{A}_{\mu}\right)_{b a} \gamma_{5}\right]+\text { H.c., } \\
\mathcal{L}_{X}=\frac{k^{\prime}}{\Lambda_{\chi}} \operatorname{Tr}\left[\bar{H}_{a} X_{b}^{\mu}\left(i D_{\mu} \mathcal{A}+i D \mathcal{A}_{\mu}\right)_{b a} \gamma_{5}\right]+\text { H.c. } \\
\mathcal{L}_{X^{\prime}}=\frac{1}{\Lambda_{\chi}{ }^{2}} \operatorname{Tr}\left[\overline { H } _ { a } X _ { b } ^ { \prime \mu \nu } \left[k_{1}\left\{D_{\mu}, D_{\nu}\right\} \mathcal{A}_{\lambda}\right.\right. \\
\left.\left.+k_{2}\left(D_{\mu} D_{\lambda} \mathcal{A}_{\nu}+D_{\nu} D_{\lambda} \mathcal{A}_{\mu}\right)\right]_{b a} \gamma^{\lambda} \gamma_{5}\right]+ \text { H.c., } \\
\mathcal{L}_{F}=\frac{1}{\Lambda_{\chi}{ }^{2}} \operatorname{Tr}\left[\overline { H } _ { a } F _ { b } ^ { \mu \nu } \left[\hat{p}_{1}\left\{D_{\mu}, D_{\nu}\right\} \mathcal{A}_{\lambda}\right.\right. \\
\left.\left.+\hat{p}_{2}\left(D_{\mu} D_{\lambda} \mathcal{A}_{\nu}+D_{\nu} D_{\lambda} \mathcal{A}_{\mu}\right)\right]_{b a} \gamma^{\lambda} \gamma_{5}\right]+ \text { H.c. },
\end{gathered}
$$

with $\bar{H}=\gamma^{0} H^{\dagger} \gamma^{0}$ and $\Lambda_{\chi}$ a scale parameter. The coupling constants $g, h, h^{\prime}, k^{\prime}, k_{1,2}, \hat{p}_{1,2}$ can be inferred from experiment, indeed bounds have been found [16]. Theoretical determinations using nonperturbative approaches are available, namely for $g$ and $h$ [28-34]. The expressions for the $\mathcal{H}_{i} \rightarrow P^{(*)} M$ decay widths, with $P^{(*)}$ in the $H$ doublet and $M$ a light pseudoscalar meson, can be found in [16], with the exception of decaying mesons in the $F$ doublet, obtained from Eq. (14) ${ }^{1}$ :

$$
\begin{aligned}
& \Gamma\left(P_{2}^{\prime *}(v, \eta) \rightarrow P(v) M\left(p_{M}\right)\right) \\
& \quad=C_{M} \frac{4 \hat{p}^{2}}{25 \pi f_{\pi}^{2} \Lambda_{\chi}^{4}} \frac{m_{P}}{m_{P_{2}^{\prime *}}}\left|\vec{p}_{M}\right|^{5}\left(m_{M}^{2}+\left|\vec{p}_{M}\right|^{2}\right), \\
& \Gamma\left(P_{2}^{\prime *}(v, \eta) \rightarrow P^{*}(v, \epsilon) M\left(p_{M}\right)\right) \\
& \quad=C_{M} \frac{8 \hat{p}^{2}}{75 \pi f_{\pi}^{2} \Lambda_{\chi}^{4}} \frac{m_{P^{*}}}{m_{P_{2}^{\prime *}}}\left|\vec{p}_{M}\right|^{5}\left(m_{M}^{2}+\left|\vec{p}_{M}\right|^{2}\right),
\end{aligned}
$$

\footnotetext{
${ }^{1}$ The expressions (15), (16) coincide with those given in $[17,18]$. Instead, our Eq. (17) is different from the one quoted in [18].
}

$$
\begin{aligned}
& \Gamma\left(P_{3}(v, \eta) \rightarrow P^{*}(v, \epsilon) M\left(p_{M}\right)\right) \\
& \quad=C_{M} \frac{4 \hat{p}^{2}}{15 \pi f_{\pi}^{2} \Lambda_{\chi}^{4}} \frac{m_{P^{*}}}{m_{P_{3}}}\left|\vec{p}_{M}\right|^{5}\left(m_{M}^{2}+\left|\vec{p}_{M}\right|^{2}\right),
\end{aligned}
$$

where $\eta(\epsilon)$ is the polarization tensor (vector), $\hat{p}$ the combination of the couplings $\hat{p}=\hat{p}_{1}+\hat{p}_{2}, \vec{p}_{M}$ the threemomentum of $M$, and the factor $C_{M}$ is different for the various mesons, $C_{\pi^{+}}=C_{K^{+}}=1, C_{\pi^{0}}=C_{K_{S}}=\frac{1}{2}$ and $C_{\eta}=\frac{2}{3}$.

\section{B. Incorporating light vector mesons}

There are several ways to incorporate the light vector mesons in the effective Lagrangian describing heavy meson decays. Here we reconsider the hidden gauge symmetry approach [35-38] applied in [19,39-41].

The hidden local symmetry method, which dates back to applications to supergravity theories $[42,43]$, exploits the equivalence of the nonlinear sigma model based on a group $G$ spontaneously broken to a subgroup $H$, to another model having $G$ as global symmetry group and $H$ as a local symmetry. This allows one to introduce the gauge bosons of the local symmetry, which are identified with the light vector mesons in applications to chiral theory. In this formulation the vector fields transform inhomogeneously under nonlinear realization of the chiral symmetry, while in alternative approaches to incorporate the vector mesons (Weinberg [44] and Callan, Coleman, Wess and Zumino [45]), the vector fields transform homogeneously. The different methods are shown to be equivalent [36,46-50].

In the hidden gauge symmetry framework one writes

$$
\Sigma=\xi_{L} \xi_{R}^{\dagger}
$$

The fields $\xi_{L, R}$ transform under $S U(3)_{L} \times S U(3)_{R} \times$ $S U(3)_{H}$ as

$$
\begin{aligned}
& \xi_{L} \rightarrow U_{L} \xi_{L} U_{H}^{\dagger}(x), \\
& \xi_{R} \rightarrow U_{R} \xi_{R} U_{H}^{\dagger}(x),
\end{aligned}
$$

where $U_{L, R} \in S U(3)_{L, R}$, and $U_{H}(x) \in S U(3)_{H}$ is a local transformation. The action of the group $S U(3)_{H}$ is hidden when one considers the field $\Sigma$. One now defines

$$
\begin{aligned}
& \mathcal{A}_{\mu}=\frac{i}{2}\left(\xi_{L}^{\dagger} \partial_{\mu} \xi_{L}-\xi_{R} \partial_{\mu} \xi_{R}^{\dagger}\right), \\
& \mathcal{V}_{\mu}=\frac{1}{2}\left(\xi_{L}^{\dagger} \partial_{\mu} \xi_{L}+\xi_{R} \partial_{\mu} \xi_{R}^{\dagger}\right) .
\end{aligned}
$$

Fixing the gauge in such a way that $\xi_{L}=\xi_{R}=\xi$, these fields can be identified with the ones in Eqs. (4) and (3). Their transformation properties under $S U(3)_{H}$ are given by Eqs. (6) and (7), identifying $U$ with $U_{H}$.

The octet of light vector mesons plays the role of gauge fields of the a hidden symmetry, and is introduced writing 


$$
\rho_{\mu}=i \frac{g_{V}}{\sqrt{2}} \hat{\rho}_{\mu}
$$

where $\hat{\rho}_{\mu}$ is a Hermitian matrix defined in analogy to the matrix $\mathcal{M}$ of pseudoscalar fields (2):

$$
\hat{\rho}_{\mu}=\left(\begin{array}{ccc}
\sqrt{\frac{1}{2}} \rho^{0}+\sqrt{\frac{1}{6}} \phi^{(8)} & \rho^{+} & K^{*+} \\
\rho^{-} & -\sqrt{\frac{1}{2}} \rho^{0}+\sqrt{\frac{1}{6}} \phi^{(8)} & K^{* 0} \\
K^{*-} & \bar{K}^{* 0} & -\sqrt{\frac{2}{3}} \phi^{(8)}
\end{array}\right)_{\mu} .
$$

The constant $g_{V}$ is set to $g_{V} \simeq 5.8$ to satisfy the KSRF relations [51,52]. The observed vector mesons $\omega$ and $\phi$ correspond to a mixing between the octet component $\phi^{(8)}$ in (22) and the singlet component $\phi^{(0)}$ :

$$
\begin{aligned}
& \phi=\sin \theta_{V} \phi^{(0)}-\cos \theta_{V} \phi^{(8)}, \\
& \omega=\cos \theta_{V} \phi^{(0)}+\sin \theta_{V} \phi^{(8)} .
\end{aligned}
$$

The angle $\theta_{V} \simeq \operatorname{ArcTan} \frac{1}{\sqrt{2}}$ realizes the ideal mixing allowing one to identify $\omega$ and $\phi$ with the flavor eigenstates $\phi_{q}=\frac{\bar{u} u+\bar{d} d}{\sqrt{2}}$ and $\phi_{s}=\bar{s} s$. In terms of these, in (22) one can replace $\frac{1}{\sqrt{3}} \phi^{(8)}=\sin \theta_{V} \phi^{(8)} \rightarrow \phi_{q}$, and $-\frac{2}{\sqrt{3}} \phi^{(8)}=$ $-\cos \theta_{V} \phi^{(8)} \rightarrow \phi_{s}:$

$\hat{\rho}_{\mu}=\left(\begin{array}{ccc}\sqrt{\frac{1}{2}} \rho^{0}+\sqrt{\frac{1}{2}} \omega & \rho^{+} & K^{*+} \\ \rho^{-} & -\sqrt{\frac{1}{2}} \rho^{0}+\sqrt{\frac{1}{2}} \omega & K^{* 0} \\ K^{*-} & \bar{K}^{* 0} & \phi\end{array}\right)_{\mu}$,

a replacement becoming exact in the large $N_{c}$ limit [53].

The antisymmetric field tensor is defined as

$$
\mathcal{F}_{\mu \nu}=\partial_{\mu} \rho_{\nu}-\partial_{\nu} \rho_{\mu}+\left[\rho_{\mu}, \rho_{\nu}\right] .
$$

$\rho_{\mu}$ transforms as $\mathcal{V}_{\mu}$ :

$$
\rho_{\mu} \rightarrow U \rho_{\mu} U^{\dagger}+U \partial_{\mu} U^{\dagger},
$$

while the difference $\mathcal{V}_{\mu}-\rho_{\mu}$, as well as $\mathcal{F}_{\mu \nu}$ transform homogenously as $\mathcal{A}_{\mu}$ :

$$
\begin{aligned}
\left(\mathcal{V}_{\mu}-\rho_{\mu}\right) & \rightarrow U\left(\mathcal{V}_{\mu}-\rho_{\mu}\right) U^{\dagger}, \\
\mathcal{F}_{\mu \nu} & \rightarrow U \mathcal{F}_{\mu \nu} U^{\dagger}
\end{aligned}
$$

The covariant derivative $D_{\alpha}$ can be defined, such that $D_{\alpha} \mathcal{F}_{\mu \nu} \rightarrow U\left(D_{\alpha} \mathcal{F}_{\mu \nu}\right) U^{\dagger}$. If $W_{\alpha}$ is a field transforming inhomogeneously, one can show that

$$
D_{\alpha} \mathcal{F}_{\mu \nu}=\partial_{\alpha} \mathcal{F}_{\mu \nu}+\left[\mathcal{F}_{\mu \nu}, W_{\alpha}\right]
$$

satisfies the previous relation. $W_{\alpha}=\mathcal{V}_{\alpha}$, or $W_{\alpha}=\rho_{\alpha}$, or a linear combination of them can be chosen, but for our purpose it is irrelevant to fix $W$, since at the leading order in the effective theory and for processes describing heavylight meson decays to another heavy one and a single light vector meson, only the partial derivative in (29) contributes to the amplitude.

\section{EFFECTIVE LAGRANGIAN TERMS AND STRONG DECAY WIDTHS}

We now construct the effective Lagrangian terms governing the decays $\mathcal{H}_{i} \rightarrow P^{(*)} V$, where $\mathcal{H}_{i}$ is a heavy-light meson, $\mathrm{V}$ a light vector meson, and $P, P^{*}$ the lowest-lying heavy-light $J^{P}=\left(0^{-}, 1^{-}\right)$mesons. For the doublets corresponding to $\ell=0$ and $\ell=1$ such Lagrangians have been derived in $[19,20]$. We denote by $\mathcal{H}^{\mu_{1} \mu_{2} \ldots \mu_{k}}$ the spin doublet which the decaying heavy meson $\mathcal{H}_{i}$ belongs to. The effective Lagrangian describing the transition $\mathcal{H}_{i} \rightarrow$ $P^{(*)} V$ can have two structures:

$$
\begin{gathered}
\mathcal{L}_{1}=-\zeta \operatorname{Tr}\left[\bar{H} \mathcal{H}^{\mu_{1} \ldots \mu_{k}} \Gamma_{\mu_{1} \ldots \mu_{k} \alpha}\left(\mathcal{V}^{\alpha}-\rho^{\alpha}\right)\right]+\text { H.c. } \\
\left.\mathcal{L}_{2}=\mu \operatorname{Tr}\left[\bar{H} \mathcal{H}^{\mu_{1} \ldots \mu_{k}} \Gamma_{\mu_{1} \ldots \mu_{k} \alpha \beta} \mathcal{F}^{\alpha \beta}\right)\right]+ \text { H.c. }
\end{gathered}
$$

with the minus sign in (30) included for later convenience. The two structures $\Gamma_{\mu_{1} \ldots \mu_{k} \alpha}$ and $\Gamma_{\mu_{1} \ldots \mu_{k} \alpha \beta}$ are chosen in such a way that the Lagrangians are invariant under heavy quark symmetry and hidden gauge symmetry transformations, parity $(\mathcal{P})$, charge conjugation $(\mathcal{C})$, and time reversal $(\mathcal{T})$. Indeed, under such discrete transformations one has

$$
\begin{array}{lcc}
\mathcal{V}^{\alpha} \stackrel{\mathcal{P}}{\rightarrow} \mathcal{V}_{\alpha} & \mathcal{V}^{\alpha} \stackrel{\mathcal{T}}{\rightarrow} \mathcal{V}_{\alpha} & \mathcal{V}^{\alpha} \stackrel{\mathcal{C}}{\rightarrow}-\left(\mathcal{V}^{\alpha}\right)^{T} \\
\rho^{\alpha} \stackrel{\mathcal{P}}{\rightarrow} \rho_{\alpha} & \rho^{\alpha} \stackrel{\mathcal{T}}{\rightarrow} \rho_{\alpha} & \rho^{\alpha} \stackrel{\mathcal{C}}{\rightarrow}-\left(\rho^{\alpha}\right)^{T} \\
\mathcal{F}^{\alpha \beta} \stackrel{\mathcal{P}}{\rightarrow} \mathcal{F}_{\alpha \beta} & \mathcal{F}^{\alpha \beta} \stackrel{\mathcal{T}}{\rightarrow}-\mathcal{F}_{\alpha \beta} \quad \mathcal{F}^{\alpha \beta} \stackrel{\mathcal{C}}{\rightarrow}-\left(\mathcal{F}^{\alpha \beta}\right)^{T},
\end{array}
$$

where $T$ means transpose. As for the heavy meson doublets, they transform under $\mathcal{P}$ and $\mathcal{T}$ as [54]

$$
\begin{aligned}
& \mathcal{H}_{v}^{\mu_{1} \ldots \mu_{k}}(x) \stackrel{\mathcal{P}}{\longrightarrow} \gamma^{0}\left(\mathcal{H}_{\bar{v}}\right)_{\mu_{1} \ldots \mu_{k}}(\bar{x}) \gamma^{0}, \\
& \mathcal{H}_{v}^{\mu_{1} \ldots \mu_{k}}(x) \stackrel{\mathcal{T}}{\longrightarrow} \hat{T}\left(\mathcal{H}_{\bar{v}}\right)_{\mu_{1} \ldots \mu_{k}}(-\bar{x}) \hat{T}^{-1},
\end{aligned}
$$

where $\bar{v}, \bar{x}$ denote the parity reflections of $v$ and $x$ (e.g., $\bar{v}^{\mu}=v_{\mu}$ ), and $\hat{T}=i \gamma^{1} \gamma^{3}$. Transforming all the fields according to these rules, it can be checked that all our effective Lagrangian terms are invariant under parity and time reversal. As for charge conjugation, discussed e.g., in [41], the effective heavy meson fields transform into the corresponding fields that contain the negative energy component of the heavy quark. For example, in the case 
of the lowest-lying doublet, denoting such field by $H_{v}^{(-)}(x)$ one has

$$
H_{v}(x) \stackrel{\mathcal{C}}{\rightarrow} \hat{C}\left(\bar{H}_{v}^{(-)}(x)\right)^{T} \hat{C}^{\dagger}
$$

where $\hat{C}=i \gamma^{2} \gamma^{0}$. Invariance under charge conjugation is obtained adding to the effective Lagrangian an antiparticle part that has the same form of the particle part except for the substitutions: $H_{v} \rightarrow H_{v}^{(-)}$and $v \rightarrow-v$. We always imply that our effective Lagrangian terms include the corresponding antiparticle parts.

Invariance under heavy quark velocity reparametrization must also be preserved [55]. The heavy quark symmetry imposes further constraints, since in the decays of the two members in a spin doublet to the ones of the lowest-lying doublet, the light meson must be emitted in the same orbital state. This reduces the number of terms in the effective Lagrangian. Beyond the leading order in the HQ expansion, additional Lagrangian terms must be included [56,57].

Considering the doublets in (1), in the effective Lagrangian terms (30) and (31) we are concerned with indices having $k=0,1,2$, that we discuss in turn. For the decay mode $P_{1} \rightarrow P_{2} V$ we have $\left|\vec{p}_{V}\right|=\frac{\lambda^{1 / 2}\left(m_{P_{1}}^{2}, m_{P_{2}}^{2}, m_{V}^{2}\right)}{2 m_{P_{1}}}$ and $E_{V}=\frac{m_{P_{1}}^{2}-m_{P_{2}}^{2}+m_{V}^{2}}{2 m_{P_{1}}}$, with $\lambda(x, y, z)=x^{2}+y^{2}+z^{2}-2 x y-$ $2 x z-2 y z$ the triangular function.

Before discussing in details the transitions of states in the various doublets, we remark that the effective Lagrangian approach is in principle applicable when the emitted light particle is soft. This is guaranteed when the mass difference between the decaying meson and the final heavy-light meson is not too large. When decays of heavier excitations are considered, it is possible that corrections from higher order terms in the effective Lagrangians could become sizeable. Nevertheless, we push our predictions also for large values of the mass of the decaying particle, considering the symmetries as the main guidelines in the description of the heavy-light meson phenomenology.

\section{A. Transitions $\tilde{\boldsymbol{H}} \rightarrow \boldsymbol{H} \boldsymbol{V}$, with $\tilde{\boldsymbol{H}}=\left(\tilde{\boldsymbol{P}}, \tilde{\boldsymbol{P}}^{*}\right)$}

When the decaying meson belongs to the $H$ doublet we have $k=0$ in Eqs. (30) and (31). Decays to $P^{(*)} V$ are not kinematically allowed for the $n=1 H$ doublet, hence we consider the radially excited $\tilde{H}$ doublet $(n=1$ is relevant for processes with intermediate virtual mesons [19]). The decays occur in $p$-wave, and the terms (30) and (31) fulfilling the constraints are

$$
\begin{gathered}
\mathcal{L}_{1}^{H}=-g_{1}^{H} \operatorname{Tr}\left[\bar{H} \tilde{H} \gamma^{\alpha}\left(\mathcal{V}_{\alpha}-\rho_{\alpha}\right)\right]+\text { H.c. }, \\
\mathcal{L}_{2}^{H}=g_{2}^{H} \frac{1}{\Lambda} \operatorname{Tr}\left[\bar{H} \tilde{H} \sigma^{\alpha \beta} \mathcal{F}_{\alpha \beta}\right]+\text { H.c. },
\end{gathered}
$$

with the parameter $\Lambda$ introduced to render the couplings dimensionless. We set $\Lambda=1 \mathrm{GeV}$. In the previous expressions, the replacement of a single $\gamma$ matrix with the four-velocity $v$ produces terms that either give the same result or vanish, a remark holding for all cases considered below. The Lagrangians (34) and (35) coincide with those obtained in [19], and from them the decay widths are worked out:

$$
\begin{aligned}
& \Gamma\left(\tilde{P}(v) \rightarrow P(v) V\left(p_{V}, \epsilon_{V}\right)\right)=C_{V} \frac{g_{V}^{2}\left(g_{1}^{H}\right)^{2}}{4 \pi m_{V}^{2}} \frac{m_{P}}{m_{\tilde{P}}}\left|\vec{p}_{V}\right|^{3}, \\
& \Gamma\left(\tilde{P}(v) \rightarrow P^{*}(v, \epsilon) V\left(p_{V}, \epsilon_{V}\right)\right)=C_{V} \frac{2 g_{V}^{2}\left(g_{2}^{H}\right)^{2}}{\pi \Lambda^{2}} \frac{m_{P^{*}}}{m_{\tilde{P}}}\left|\vec{p}_{V}\right|^{3},
\end{aligned}
$$

$\Gamma\left(\tilde{P}^{*}(v, \tilde{\epsilon}) \rightarrow P(v) V\left(p_{V}, \epsilon_{V}\right)\right)=C_{V} \frac{2 g_{V}^{2}\left(g_{2}^{H}\right)^{2}}{3 \pi \Lambda^{2}} \frac{m_{P}}{m_{\tilde{P}^{*}}}\left|\vec{p}_{V}\right|^{3}$,

$$
\begin{aligned}
& \Gamma\left(\tilde{P}^{*}(v, \tilde{\epsilon}) \rightarrow P^{*}(v, \epsilon) V\left(p_{V}, \epsilon_{V}\right)\right) \\
& \quad=C_{V} \frac{g_{V}^{2}}{12 \pi m_{V}^{2}} \frac{m_{P^{*}}}{m_{\tilde{P}^{*}}}\left(16 \tilde{m}_{V}^{2}\left(g_{2}^{H}\right)^{2}+3\left(g_{1}^{H}\right)^{2}\right)\left|\vec{p}_{V}\right|^{3},
\end{aligned}
$$

with $\epsilon_{V}$ and $\epsilon, \tilde{\epsilon}$ light and heavy meson polarization vectors, $\tilde{m}_{V}=\frac{m_{V}}{\Lambda}$, and $C_{V}=1$ for $V=\rho^{ \pm}, K^{* \pm}, K^{* 0}, \bar{K}^{* 0}, \varphi, C_{V}=$ $\frac{1}{2}$ for $V=\rho^{0}, \omega$.

Relations among the decay widths, not involving the coupling constants, can be constructed:

$$
\begin{aligned}
& R_{H}=\frac{\Gamma\left(\tilde{P} \rightarrow P^{*} V\right)}{\Gamma\left(\tilde{P}^{*} \rightarrow P V\right)}=3 \frac{m_{P^{*}}}{m_{P}} \frac{m_{\tilde{P}^{*}}^{4}}{m_{\tilde{P}}^{4}} \frac{\lambda^{3 / 2}\left(m_{\tilde{P}}^{2}, m_{P^{*}}^{2}, m_{V}^{2}\right)}{\lambda^{3 / 2}\left(m_{\tilde{P}^{*}}^{2}, m_{P}^{2}, m_{V}^{2}\right)}, \quad \\
& \Gamma\left(\tilde{P}^{*} \rightarrow P^{*} V\right)= \frac{m_{P^{*}}}{m_{P}}\left\{2 \frac{\lambda^{3 / 2}\left(m_{\tilde{P}^{*}}^{2}, m_{P^{*}}^{2}, m_{V}^{2}\right)}{\lambda^{3 / 2}\left(m_{\tilde{P}^{*}}^{2}, m_{P}^{2}, m_{V}^{2}\right)} \Gamma\left(\tilde{P}^{*} \rightarrow P V\right)\right. \\
&\left.+\frac{m_{\tilde{P}}^{4}}{m_{\tilde{P}^{*}}^{4}} \frac{\lambda^{3 / 2}\left(m_{\tilde{P}^{*}}^{2}, m_{P^{*}}^{2}, m_{V}^{2}\right)}{\lambda^{3 / 2}\left(m_{\tilde{P}}^{2}, m_{P}^{2}, m_{V}^{2}\right)} \Gamma(\tilde{P} \rightarrow P V)\right\} .
\end{aligned}
$$

Other relations independent of the couplings can be worked out considering modes with different final light vector mesons, as discussed in Sec. IV.

$$
\text { B. } \tilde{S} \rightarrow H V \text {, with } \tilde{S}=\left(\tilde{P}_{0}^{*}, \tilde{P}_{1}^{\prime}\right)
$$

When the decaying meson belongs to the $S$ doublet one has $k=0$ in Eqs. (30) and (31). The $P^{(*)} V$ phase space is closed for $n=1$; therefore, we consider radial excitations in the $\tilde{S}$ doublet. The transitions occur in $s$-wave, and the effective Lagrangian terms (30) and (31) read

$$
\begin{gathered}
\mathcal{L}_{1}^{S}=-g_{1}^{S} \operatorname{Tr}\left[\bar{H} \tilde{S} \gamma^{\alpha}\left(\mathcal{V}_{\alpha}-\rho_{\alpha}\right)\right]+\text { H.c. }, \\
\mathcal{L}_{2}^{S}=g_{2}^{S} \frac{1}{\Lambda} \operatorname{Tr}\left[\bar{H} \tilde{S} \sigma^{\alpha \beta} \mathcal{F}_{\alpha \beta}\right]+\text { H.c. }
\end{gathered}
$$


as also obtained in [20]. The decay widths read

$$
\begin{aligned}
& \Gamma\left(\tilde{P}_{0}^{*}(v) \rightarrow P^{*}(v, \epsilon) V\left(p_{V}, \epsilon_{V}\right)\right) \\
& \quad=C_{V} \frac{g_{V}^{2}}{4 \pi m_{V}^{2}} \frac{m_{P^{*}}}{m_{\tilde{P}_{0}^{*}}^{*}}\left\{\left(g_{1}^{S}\right)^{2}\left(3 m_{V}^{2}+\left|\vec{p}_{V}\right|^{2}\right)+12 g_{1}^{S} g_{2}^{S} \tilde{m}_{V} m_{V} \sqrt{m_{V}^{2}+\left|\vec{p}_{V}\right|^{2}}+4\left(g_{2}^{S}\right)^{2} \tilde{m}_{V}^{2}\left(3 m_{V}^{2}+2\left|\vec{p}_{V}\right|^{2}\right)\right\}\left|\vec{p}_{V}\right|, \\
& \Gamma\left(\tilde{P}_{1}^{\prime}(v, \eta) \rightarrow P(v) V\left(p_{V}, \epsilon_{V}\right)\right) \\
& \quad=C_{V} \frac{g_{V}^{2}}{12 \pi m_{V}^{2}} \frac{m_{P}}{m_{\tilde{P}_{1}^{\prime}}}\left\{\left(g_{1}^{S}\right)^{2}\left(3 m_{V}^{2}+\left|\vec{p}_{V}\right|^{2}\right)+12 g_{1}^{S} g_{2}^{S} \tilde{m}_{V} m_{V} \sqrt{m_{V}^{2}+\left|\vec{p}_{V}\right|^{2}}+4\left(g_{2}^{S}\right)^{2} \tilde{m}_{V}^{2}\left(3 m_{V}^{2}+2\left|\vec{p}_{V}\right|^{2}\right)\right\}\left|\vec{p}_{V}\right|, \\
& \Gamma\left(\tilde{P}_{1}^{\prime}(v, \eta) \rightarrow P^{*}(v, \epsilon) V\left(p_{V}, \epsilon_{V}\right)\right) \\
& \quad=C_{V} \frac{g_{V}^{2}}{6 \pi m_{V}^{2}} \frac{m_{P^{*}}}{m_{\tilde{P}_{1}^{\prime}}^{\prime}}\left\{\left(g_{1}^{S}\right)^{2}\left(3 m_{V}^{2}+\left|\vec{p}_{V}\right|^{2}\right)+12 g_{1}^{S} g_{2}^{S} \tilde{m}_{V} m_{V} \sqrt{m_{V}^{2}+\left|\vec{p}_{V}\right|^{2}}+4\left(g_{2}^{S}\right)^{2} \tilde{m}_{V}^{2}\left(3 m_{V}^{2}+2\left|\vec{p}_{V}\right|^{2}\right)\right\}\left|\vec{p}_{V}\right|,
\end{aligned}
$$

with $\eta$ polarization vector. The transition $P_{0}^{*}(v) \rightarrow$ $P(v) V\left(p_{V}, \epsilon_{V}\right)$ is forbidden.

$$
\text { C. } \tilde{T} \rightarrow H V \text {, with } \tilde{T}=\left(\tilde{\boldsymbol{P}}_{1}, \tilde{\boldsymbol{P}}_{2}^{*}\right)
$$

For the decays of the states in the $T$ doublet one has $k=1$ in (30) and (31). The transitions to $P^{(*)} V$ is not kinematically allowed for the $n=1 T$ doublet, hence consider $n=2 \tilde{T}$. The transitions proceed in $d$-wave, and the effective Lagrangian reads

$$
\mathcal{L}_{2}^{T}=i h^{T} \frac{1}{\Lambda^{2}} \operatorname{Tr}\left[\bar{H} T_{\mu} \sigma^{\alpha \beta} \mathcal{D}^{\mu} \mathcal{F}_{\alpha \beta}\right]+\text { H.c. }
$$

with the covariant derivative acting on the light vector meson field tensor. The resulting decay widths are

$\Gamma\left(\tilde{P}_{1}(v, \eta) \rightarrow P(v) V\left(p_{V}, \epsilon_{V}\right)\right)=C_{V} \frac{g_{V}^{2}\left(h^{T}\right)^{2}}{9 \pi \Lambda^{4}} \frac{m_{P}}{m_{\tilde{P}_{1}}}\left|\vec{p}_{V}\right|^{5}$,

$\Gamma\left(\tilde{P}_{1}(v, \eta) \rightarrow P^{*}(v, \epsilon) V\left(p_{V}, \epsilon_{V}\right)\right)=C_{V} \frac{5 g_{V}^{2}\left(h^{T}\right)^{2}}{9 \pi \Lambda^{4}} \frac{m_{P^{*}}}{m_{\tilde{P}_{1}}}\left|\vec{p}_{V}\right|^{5}$,

$\Gamma\left(\tilde{P}_{2}^{*}(v, \eta) \rightarrow P(v) V\left(p_{V}, \epsilon_{V}\right)\right)=C_{V} \frac{g_{V}^{2}\left(h^{T}\right)^{2}}{5 \pi \Lambda^{4}} \frac{m_{P}}{m_{\tilde{P}_{2}^{*}}}\left|\vec{p}_{V}\right|^{5}$,

$\Gamma\left(\tilde{P}_{2}^{*}(v, \eta) \rightarrow P^{*}(v, \epsilon) V\left(p_{V}, \epsilon_{V}\right)\right)=C_{V} \frac{7 g_{V}^{2}\left(h^{T}\right)^{2}}{15 \pi \Lambda^{4}} \frac{m_{P^{*}}}{m_{\tilde{P}_{2}^{*}}}\left|\vec{p}_{V}\right|^{5}$
The relations are fulfilled:

$$
\begin{aligned}
& \frac{\Gamma\left(\tilde{P}_{1} \rightarrow P^{*} V\right)}{\Gamma\left(\tilde{P}_{1} \rightarrow P V\right)}=5 \frac{m_{P^{*}}}{m_{P}} \frac{\left(\left|\vec{p}_{V}\right|^{\left(\tilde{P}_{1} \rightarrow P^{*} V\right)}\right)^{5}}{\left(\left|\vec{p}_{V}\right|^{\left(\tilde{P}_{1} \rightarrow P V\right)}\right)^{5}}, \\
& \frac{\Gamma\left(\tilde{P}_{2}^{*} \rightarrow P^{*} V\right)}{\Gamma\left(\tilde{P}_{2}^{*} \rightarrow P V\right)}=\frac{7}{3} \frac{m_{P^{*}}}{m_{P}} \frac{\left(\left|\vec{p}_{V}\right|^{\left(\tilde{P}_{2}^{*} \rightarrow P^{*} V\right)}\right)^{5}}{\left(\left|\vec{p}_{V}\right|^{\left(\tilde{P}_{2}^{*} \rightarrow P V\right)}\right)^{5}} .
\end{aligned}
$$

Ratios of decay rates for modes with different final light vector mesons, independent of the coupling constant, will be constructed below.

$$
\text { D. } X \rightarrow H V \text {, with } X=\left(P_{1}^{*}, P_{2}\right)
$$

For $X$ doublet one has $k=1$ in (30) and (31). No candidates belonging to such a doublet have been observed, and we do not know whether the $P^{(*)} V$ channels are open for the $n=1$ states. The transitions occur in $p$-wave and are governed by the Lagrangian

$$
\mathcal{L}_{2}^{X}=i h^{X} \frac{1}{\Lambda^{2}} \operatorname{Tr}\left[\bar{H} X_{\mu} \sigma^{\alpha \beta} \mathcal{D}^{\mu} \mathcal{F}_{\alpha \beta}\right]+\text { H.c. }
$$

with the covariant derivative acting on the light vector meson field tensor. The decay widths are given by

$$
\begin{aligned}
& \Gamma\left(P_{1}^{*}(v, \eta) \rightarrow P(v) V\left(p_{V}, \epsilon_{V}\right)\right) \\
& \quad=C_{V} \frac{g_{V}^{2}\left(h^{X}\right)^{2}}{9 \pi \Lambda^{4}} \frac{m_{P}}{m_{P_{1}^{*}}}\left|\vec{p}_{V}\right|^{3}\left(m_{V}^{2}+\left|\vec{p}_{V}\right|^{2}\right), \\
& \Gamma\left(P_{1}^{*}(v, \eta) \rightarrow P^{*}(v, \epsilon) V\left(p_{V}, \epsilon_{V}\right)\right) \\
& \quad=C_{V} \frac{g_{V}^{2}\left(h^{X}\right)^{2}}{9 \pi \Lambda^{4}} \frac{m_{P^{*}}}{m_{P_{1}^{*}}}\left|\vec{p}_{V}\right|^{3}\left(8 m_{V}^{2}+5\left|\vec{p}_{V}\right|^{2}\right),
\end{aligned}
$$




$$
\begin{gathered}
\Gamma\left(P_{2}(v, \eta) \rightarrow P(v) V\left(p_{V}, \epsilon_{V}\right)\right) \\
=C_{V} \frac{g_{V}^{2}\left(h^{X}\right)^{2}}{15 \pi \Lambda^{4}} \frac{m_{P}}{m_{P_{2}}}\left|\vec{p}_{V}\right|^{3}\left(5 m_{V}^{2}+3\left|\vec{p}_{V}\right|^{2}\right), \\
\Gamma\left(P_{2}(v, \eta) \rightarrow P^{*}(v, \epsilon) V\left(p_{V}, \epsilon_{V}\right)\right) \\
=C_{V} \frac{g_{V}^{2}\left(h^{X}\right)^{2}}{15 \pi \Lambda^{4}} \frac{m_{P^{*}}}{m_{P_{2}}}\left|\vec{p}_{V}\right|^{3}\left(10 m_{V}^{2}+7\left|\vec{p}_{V}\right|^{2}\right) .
\end{gathered}
$$

Coupling-independent ratios of decay widths are

$$
\begin{aligned}
& \frac{\Gamma\left(P_{1}^{*} \rightarrow P^{*} V\right)}{\Gamma\left(P_{1}^{*} \rightarrow P V\right)} \\
& \quad=\frac{m_{P^{*}}}{m_{P}} \frac{\left(\left|\vec{p}_{V}\right|^{\left(P_{1}^{*} \rightarrow P^{*} V\right)}\right)^{3}}{\left(\left|\vec{p}_{V}\right|^{\left(P_{1}^{*} \rightarrow P V\right)}\right)^{3}} \frac{8 m_{V}^{2}+5\left(\left|\vec{p}_{V}\right|^{\left(P_{1}^{*} \rightarrow P^{*} V\right)}\right)^{2}}{m_{V}^{2}+\left(\left|\vec{p}_{V}\right|^{\left(P_{1}^{*} \rightarrow P V\right)}\right)^{2}}, \\
& \frac{\Gamma\left(P_{2} \rightarrow P^{*} V\right)}{\Gamma\left(P_{2} \rightarrow P V\right)} \\
& \quad=\frac{m_{P^{*}}}{m_{P}} \frac{\left(\left|\vec{p}_{V}\right|^{\left(P_{2} \rightarrow P^{*} V\right)}\right)^{3}}{\left(\left|\vec{p}_{V}\right|^{\left(P_{2} \rightarrow P V\right)}\right)^{3}} \frac{10 m_{V}^{2}+7\left(\left|\vec{p}_{V}\right|^{\left(P_{2} \rightarrow P^{*} V\right)}\right)^{2}}{5 m_{V}^{2}+3\left(\left|\vec{p}_{V}\right|^{\left(P_{2} \rightarrow P V\right)}\right)^{2}},
\end{aligned}
$$

while ratios of decay widths for processes with different final light vector meson are discussed in Sec. IV.

$$
\text { E. } X^{\prime} \rightarrow H V \text {, with } X^{\prime}=\left(P_{2}^{\prime}, P_{3}^{*}\right)
$$

For the decays of the members of the $X^{\prime}$ doublet one has to consider $k=2$ in (30) and (31). The processes occur in $f$-wave, with Lagrangian

$$
\mathcal{L}_{2}^{X^{\prime}}=k^{X^{\prime}} \frac{1}{\Lambda^{3}} \operatorname{Tr}\left[\bar{H} X_{\mu \nu}^{\prime} \mathcal{D}^{\mu} \mathcal{D}^{\nu} \sigma^{\alpha \beta} \mathcal{F}_{\alpha \beta}\right]+\text { H.c. }
$$

The decay widths read

$$
\begin{aligned}
& \Gamma\left(P_{2}^{\prime}(v, \eta) \rightarrow P(v) V\left(p_{V}, \epsilon_{V}\right)\right)=C_{V} \frac{4 g_{V}^{2}\left(k^{X^{\prime}}\right)^{2}}{75 \pi \Lambda^{6}} \frac{m_{P}}{m_{P_{2}^{\prime}}}\left|\vec{p}_{V}\right|^{7}, \\
& \Gamma\left(P_{2}^{\prime}(v, \eta) \rightarrow P^{*}(v, \epsilon) V\left(p_{V}, \epsilon_{V}\right)\right)=C_{V} \frac{16 g_{V}^{2}\left(k^{X^{\prime}}\right)^{2}}{75 \pi \Lambda^{6}} \frac{m_{P}^{*}}{m_{P_{2}^{\prime}}}\left|\vec{p}_{V}\right|^{7},
\end{aligned}
$$

$$
\Gamma\left(P_{3}^{*}(v, \eta) \rightarrow P(v) V\left(p_{V}, \epsilon_{V}\right)\right)=C_{V} \frac{8 g_{V}^{2}\left(k^{X^{\prime}}\right)^{2}}{105 \pi \Lambda^{6}} \frac{m_{P}}{m_{P_{3}^{*}}}\left|\vec{p}_{V}\right|^{7}
$$

$$
\begin{gathered}
\Gamma\left(P_{3}^{*}(v, \eta) \rightarrow P^{*}(v, \epsilon) V\left(p_{V}, \epsilon_{V}\right)\right) \\
=C_{V} \frac{4 g_{V}^{2}\left(k^{X^{\prime}}\right)^{2}}{21 \pi \Lambda^{6}} \frac{m_{P}^{*}}{m_{P_{3}^{*}}}\left|\vec{p}_{V}\right|^{7} .
\end{gathered}
$$

For this doublet the relations are fulfilled:

$$
\begin{aligned}
& \frac{\Gamma\left(P_{2}^{\prime} \rightarrow P^{*} V\right)}{\Gamma\left(P_{2}^{\prime} \rightarrow P V\right)}=4 \frac{m_{P^{*}}}{m_{P}} \frac{\left(\left|\vec{p}_{V}\right|^{\left(P_{2}^{\prime} \rightarrow P^{*} V\right)}\right)^{7}}{\left(\left|\vec{p}_{V}\right|^{\left(P_{2}^{\prime} \rightarrow P V\right)}\right)^{7}} \\
& \frac{\Gamma\left(P_{3}^{*} \rightarrow P^{*} V\right)}{\Gamma\left(P_{3}^{*} \rightarrow P V\right)}=\frac{5}{2} \frac{m_{P^{*}}}{m_{P}} \frac{\left(\left|\vec{p}_{V}\right|^{\left(P_{3}^{*} \rightarrow P^{*} V\right)}\right)^{7}}{\left(\left|\vec{p}_{V}\right|^{\left(P_{3}^{*} \rightarrow P V\right)}\right)^{7}}
\end{aligned}
$$

$$
\text { F. } \boldsymbol{F} \rightarrow \boldsymbol{H} \boldsymbol{V}, \text { with } \boldsymbol{F}=\left(\boldsymbol{P}_{2}^{*}, \boldsymbol{P}_{\mathbf{3}}\right)
$$

The case of the $F$ doublet requires $k=2$ in (30) and (31). The transitions occur in $d$-wave with effective Lagrangian

$$
\mathcal{L}_{2}^{F}=k_{1}^{F} \frac{1}{\Lambda^{2}} \operatorname{Tr}\left[\bar{H} F_{\mu \nu}\left(g^{\mu \alpha} \mathcal{D}^{\nu} \gamma^{\beta}+g^{\nu \alpha} \mathcal{D}^{\mu} \gamma^{\beta}-g^{\mu \beta} \mathcal{D}^{\nu} \gamma^{\alpha}-g^{\nu \beta} \mathcal{D}^{\mu} \gamma^{\alpha}\right) \mathcal{F}_{\alpha \beta}\right]+k_{2}^{F} \frac{1}{\Lambda^{3}} \operatorname{Tr}\left[\bar{H} F_{\mu \nu} \mathcal{D}^{\mu} \mathcal{D}^{\nu} \sigma^{\alpha \beta} \mathcal{F}_{\alpha \beta}\right]+\text { H.c. }
$$

and decay widths

$$
\begin{gathered}
\Gamma\left(P_{2}^{* *}(v, \eta) \rightarrow P(v) V\left(p_{V}, \epsilon_{V}\right)\right)=C_{V} \frac{4 g_{V}^{2}}{75 \pi \Lambda^{4}} \frac{m_{P}}{m_{P_{2}^{*}}}\left|\vec{p}_{V}\right|^{5}\left(3 k_{1}^{F}+\frac{k_{2}^{F}}{\Lambda} \sqrt{m_{V}^{2}+\left|\vec{p}_{V}\right|^{2}}\right)^{2}, \quad(69) \\
\Gamma\left(P_{2}^{\prime *}(v, \eta) \rightarrow P^{*}(v, \epsilon) V\left(p_{V}, \epsilon_{V}\right)\right)=C_{V} \frac{2 g_{V}^{2}}{75 \pi \Lambda^{4}} \frac{m_{P^{*}}}{m_{P_{2}^{*}}}\left|\vec{p}_{V}\right|^{5}\left\{12\left(k_{1}^{F}\right)^{2}+\frac{8}{\Lambda} k_{1}^{F} k_{2}^{F} \sqrt{\left.m_{V}^{2}+\left|\vec{p}_{V}\right|^{2}+\left(\frac{k_{2}^{F}}{\Lambda}\right)^{2}\left(13 m_{V}^{2}+8\left|\vec{p}_{V}\right|^{2}\right)\right\},}\right. \\
\Gamma\left(P_{3}(v, \eta) \rightarrow P(v) V\left(p_{V}, \epsilon_{V}\right)\right)=C_{V} \frac{2 g_{V}^{2}}{105 \pi \Lambda^{6}} \frac{m_{P}}{m_{P_{3}}}\left(k_{2}^{F}\right)^{2}\left|\vec{p}_{V}\right|^{5}\left(7 m_{V}^{2}+4\left|\vec{p}_{V}\right|^{2}\right), \\
\Gamma\left(P_{3}(v, \eta) \rightarrow P^{*}(v, \epsilon) V\left(p_{V}, \epsilon_{V}\right)\right)=C_{V} \frac{4 g_{V}^{2}}{105 \pi \Lambda^{4}} \frac{m_{P^{*}}}{m_{P_{3}}}\left|\vec{p}_{V}\right|^{5}\left\{21\left(k_{1}^{F}\right)^{2}+\frac{14}{\Lambda} k_{1}^{F} k_{2}^{F} \sqrt{\left.m_{V}^{2}+\left|\vec{p}_{V}\right|^{2}+\left(\frac{k_{2}^{F}}{\Lambda}\right)^{2}\left(7 m_{V}^{2}+5\left|\vec{p}_{V}\right|^{2}\right)\right\} .}\right.
\end{gathered}
$$


A relation independent of the couplings connects various modes:

$\Gamma\left(P_{3} \rightarrow P^{*} V\right)=\frac{\left(\left|\vec{p}_{V}\right|^{\left(P_{3} \rightarrow P^{*} V\right)}\right)^{5}}{\left(E_{V}^{\left(P_{2}^{*} \rightarrow P V\right)}-E_{V}^{\left(P_{2}^{*} \rightarrow P^{*} V\right)}\right)}\left\{C_{1}^{\left(P_{2}^{*} \rightarrow P^{*} V\right)} \Gamma\left(P_{2}^{\prime *} \rightarrow P^{*} V\right)+C_{2}^{\left(P_{2}^{* *} \rightarrow P^{*} V\right)} \Gamma\left(P_{2}^{* *} \rightarrow P V\right)+C_{3}^{\left(P_{2}^{\prime *} \rightarrow P^{*} V\right)} \Gamma\left(P_{3} \rightarrow P V\right)\right\}$,

where

$$
\begin{aligned}
C_{1}^{\left(P_{2}^{\prime *} \rightarrow P^{*} V\right)}= & \frac{5}{2} \frac{m_{P_{2}^{\prime *}}}{m_{P_{3}}} \frac{E_{V}^{\left(P_{2}^{\prime *} \rightarrow P V\right)}-E_{V}^{\left(P_{3} \rightarrow P^{*} V\right)}}{\left(\left|\vec{p}_{V}\right|^{\left(P_{2}^{\prime *} \rightarrow P^{*} V\right)}\right)^{5}} \\
C_{2}^{\left(P_{2}^{\prime *} \rightarrow P^{*} V\right)}= & \frac{5}{3} \frac{m_{P_{2}^{\prime *}}}{m_{P_{3}}} \frac{m_{P^{*}}}{m_{P}} \frac{E_{V}^{\left(P_{3} \rightarrow P^{*} V\right)}-E_{V}^{\left(P_{2}^{* *} \rightarrow P^{*} V\right)}}{\left(\left|\vec{p}_{V}\right|^{\left(P_{2}^{* *} \rightarrow P V\right)}\right)^{5}} \\
C_{3}^{\left(P_{2}^{\prime *} \rightarrow P^{*} V\right)}= & \frac{m_{P^{*}}}{m_{P_{3}}} \frac{1}{\left(\left|\vec{p}_{V}\right|^{\left(P_{3} \rightarrow P V\right)}\right)^{5}} \frac{1}{7 m_{V}^{2}+4\left(\left|\vec{p}_{V}\right|^{\left(P_{3} \rightarrow P V\right)}\right)^{2}}\left\{28\left(\left|\vec{p}_{V}\right|^{\left(P_{2}^{* *} \rightarrow P^{*} V\right)}\right)^{2}\left(E_{V}^{\left(P_{3} \rightarrow P^{*} V\right)}-E_{V}^{\left(P_{2}^{\prime *} \rightarrow P V\right)}\right)\right. \\
& +\frac{14}{3}\left(\left|\vec{p}_{V}\right|^{\left(P_{2}^{* *} \rightarrow P V\right)}\right)^{2}\left(E_{V}^{\left(P_{2}^{\prime *} \rightarrow P^{*} V\right)}-E_{V}^{\left(P_{3} \rightarrow P^{*} V\right)}\right)+10\left(\left|\vec{p}_{V}\right|^{\left(P_{3} \rightarrow P^{*} V\right)}\right)^{2}\left(E_{V}^{\left(P_{2}^{\prime *} \rightarrow P V\right)}-E_{V}^{\left(P_{2}^{\prime *} \rightarrow P^{*} V\right)}\right) \\
& \left.-\frac{7}{6} m_{V}^{2}\left(27 E_{V}^{\left(P_{2}^{\prime *} \rightarrow P V\right)}+8 E_{V}^{\left(P_{2}^{\prime *} \rightarrow P^{*} V\right)}-35 E_{V}^{\left(P_{3} \rightarrow P^{*} V\right)}\right)\right\} .
\end{aligned}
$$

Moreover, for $V_{A}$ and $V_{B}$ two light vector mesons one simply has

$$
R_{V_{A} V_{B}}^{P_{3}}=\frac{\Gamma\left(P_{3} \rightarrow P_{A} V_{A}\right)}{\Gamma\left(P_{3} \rightarrow P_{B} V_{B}\right)}=\frac{C_{V_{A}}}{C_{V_{B}}} \frac{\left|\vec{p}_{V_{A}}\right|^{5}\left(7 m_{V_{A}}^{2}+4\left|\vec{p}_{V_{A}}\right|^{2}\right)}{\left|\vec{p}_{V_{B}}\right|^{5}\left(7 m_{V_{B}}^{2}+4\left|\vec{p}_{V_{B}}\right|^{2}\right)}
$$

\section{NUMERICAL ANALYSIS: CHARM}

The expressions in the previous section allow one to construct quantities useful for the classification of high mass charmed and beauty states. In Table VI we collect the observed $h \bar{q}, h \bar{s}$ mesons, with $q=u, d$ and $h=c, b$. For the various $\mathcal{H}_{i} \rightarrow P^{(*)} V$ modes, ratios of decay widths of $\mathcal{H}_{i}$ to different light vector mesons, and ratios of decay widths involving the same light vector meson and a different member of the final $H$ doublet can be constructed, obtaining quantities independent of the coupling constants in the effective Lagrangians. For nonstrange decaying mesons we define

\begin{tabular}{|c|c|c|c|c|c|c|c|c|c|c|}
\hline Doublet & $s_{\ell}^{P}$ & $J^{P}$ & $c \bar{q}(n=1)$ & $c \bar{q}(n=2)$ & $c \bar{s}(n=1)$ & $c \bar{s}(n=2)$ & $b \bar{q}(n=1)$ & $b \bar{q}(n=2)$ & $b \bar{s}(n=1)$ & $b \bar{s}(n=2)$ \\
\hline \multirow[t]{2}{*}{$H$} & $\frac{1}{2}-^{-}$ & $0^{-}$ & $D(1869)$ & $\mathrm{D}(\mathbf{2 5 5 0 )}$ & $D_{s}(1968)$ & & $B(5279)$ & $\mathbf{B}_{\mathbf{J}}(\mathbf{5 8 4 0})$ & $B_{s}(5366)$ & \\
\hline & & $1^{-}$ & $D^{*}(2010)$ & $\mathbf{D}^{*}(\mathbf{2 6 0 0})$ & $D_{s}^{*}(2112)$ & $D_{s 1}^{*}(2700)$ & $B^{*}(5325)$ & $\mathbf{B}_{\mathbf{J}}(\mathbf{5 9 6 0})$ & $B_{s}^{*}(5415)$ & \\
\hline \multirow[t]{2}{*}{$S$} & $\frac{1}{2}+$ & $0^{+}$ & $D_{0}^{*}(2400)$ & & $D_{s 0}^{*}(2317)$ & & & & & \\
\hline & & $1^{+}$ & $D_{1}^{\prime}(2430)$ & & $D_{s 1}^{\prime}(2460)$ & & & & & \\
\hline \multirow[t]{2}{*}{$T$} & $\frac{3}{2}+$ & $1^{+}$ & $D_{1}(2420)$ & & $D_{s 1}(2536)$ & & $B_{1}(5721)$ & & $B_{s 1}(5830)$ & \\
\hline & & $2^{+}$ & $D_{2}^{*}(2460)$ & $\mathrm{D}_{2}^{*}(\mathbf{3 0 0 0})$ & $D_{s 2}^{*}(2573)$ & & $B_{2}^{*}(5747)$ & & $B_{s 2}^{*}(5840)$ & \\
\hline$X$ & $\frac{3}{2}^{-}$ & $\begin{array}{l}1^{-} \\
2^{-}\end{array}$ & $\mathbf{D}_{\mathbf{J}}^{*}(\mathbf{2 7 6 0})$ & & $\mathrm{D}_{\mathrm{s} 1}^{*}(\mathbf{2 8 6 0})$ & & & & & \\
\hline \multirow[t]{2}{*}{$X^{\prime}$} & $\frac{5}{2}-$ & $2^{-}$ & $D_{2}^{\prime}(2740)$ & & & & & & & \\
\hline & & $3^{-}$ & $D_{3}^{*}(2760)$ & & $D_{s 3}^{*}(2860)$ & & & & & \\
\hline$F$ & $\frac{5}{2}+$ & $\begin{array}{l}2^{+} \\
3^{+}\end{array}$ & $\mathbf{D}_{2}^{*}(\mathbf{3 0 0 0})$ & & & & & & & \\
\hline
\end{tabular}

$$
\begin{aligned}
R_{\omega \rho}^{\mathcal{H}_{i}^{+} \rightarrow D} & =\frac{\Gamma\left(\mathcal{H}_{i}^{+} \rightarrow D^{+} \omega\right)}{\Gamma\left(\mathcal{H}_{i}^{+} \rightarrow D^{+} \rho^{0}\right)+\Gamma\left(\mathcal{H}_{i}^{+} \rightarrow D^{0} \rho^{+}\right)}, \\
R_{K^{*} \rho}^{\mathcal{H}_{+}^{+} \rightarrow D_{(s)}} & =\frac{\Gamma\left(\mathcal{H}_{i}^{+} \rightarrow D_{s} \bar{K}^{* 0}\right)}{\Gamma\left(\mathcal{H}_{i}^{+} \rightarrow D^{+} \rho^{0}\right)+\Gamma\left(\mathcal{H}_{i}^{+} \rightarrow D^{0} \rho^{+}\right)},
\end{aligned}
$$

TABLE VI. Observed mesons with open charm and open beauty, classified in HQ doublets. The assignment for the states in boldface is uncertain. Two possible classifications are indicated for $D_{2}^{*}(3000)$. 

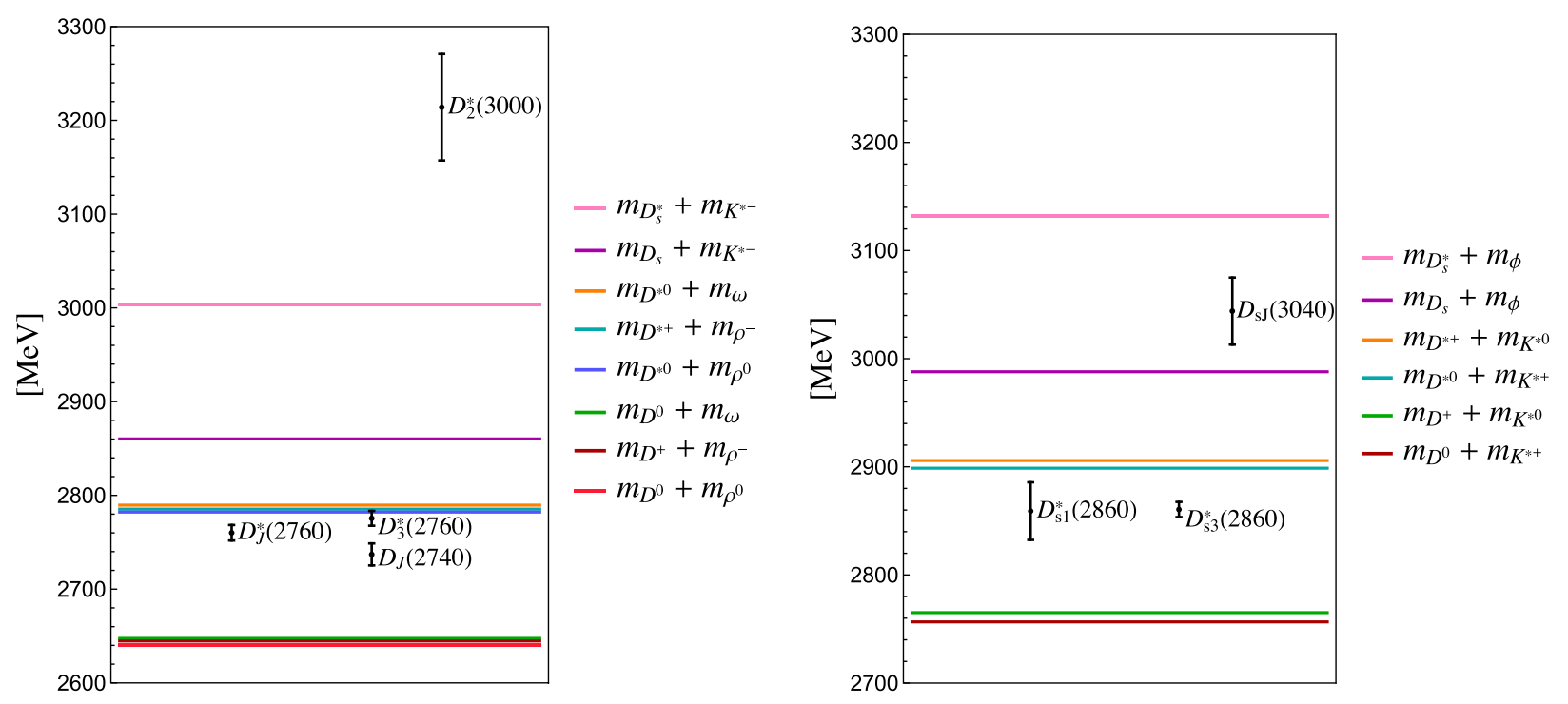

FIG. 1. $\quad P V$ and $P^{*} V$ thresholds for decaying neutral nonstrange (left) and for strange charmed mesons (right). The position of several resonances, with the mass uncertainty, is indicated.

$$
\begin{aligned}
R_{\omega \rho}^{\mathcal{H}_{i}^{+} \rightarrow D^{*}} & =\frac{\Gamma\left(\mathcal{H}_{i}^{+} \rightarrow D^{*+} \omega\right)}{\Gamma\left(\mathcal{H}_{i}^{+} \rightarrow D^{*+} \rho^{0}\right)+\Gamma\left(\mathcal{H}_{i}^{+} \rightarrow D^{* 0} \rho^{+}\right)}, \\
R_{K^{*} \rho}^{\mathcal{H}_{i}^{+} \rightarrow D_{(s)}^{*}} & =\frac{\Gamma\left(\mathcal{H}_{i}^{+} \rightarrow D_{s}^{*} \bar{K}^{* 0}\right)}{\Gamma\left(\mathcal{H}_{i}^{+} \rightarrow D^{*+} \rho^{0}\right)+\Gamma\left(\mathcal{H}_{i}^{+} \rightarrow D^{* 0} \rho^{+}\right)},
\end{aligned}
$$

and

$$
\begin{aligned}
R_{\omega \rho}^{\mathcal{H}_{i}^{0} \rightarrow D} & =\frac{\Gamma\left(\mathcal{H}_{i}^{0} \rightarrow D^{0} \omega\right)}{\Gamma\left(\mathcal{H}_{i}^{0} \rightarrow D^{0} \rho^{0}\right)+\Gamma\left(\mathcal{H}_{i}^{0} \rightarrow D^{+} \rho^{-}\right)}, \\
R_{K_{i}^{*} \rho}^{\mathcal{H}_{0}^{0} \rightarrow D_{(s)}} & =\frac{\Gamma\left(\mathcal{H}_{i}^{0} \rightarrow D_{s} K^{*-}\right)}{\Gamma\left(\mathcal{H}_{i}^{0} \rightarrow D^{0} \rho^{0}\right)+\Gamma\left(\mathcal{H}_{i}^{0} \rightarrow D^{+} \rho^{-}\right)}, \\
R_{\omega \rho}^{\mathcal{H}_{i}^{0} \rightarrow D^{*}} & =\frac{\Gamma\left(\mathcal{H}_{i}^{0} \rightarrow D^{* 0} \omega\right)}{\Gamma\left(\mathcal{H}_{i}^{0} \rightarrow D^{* 0} \rho^{0}\right)+\Gamma\left(\mathcal{H}_{i}^{0} \rightarrow D^{*+} \rho^{-}\right)}, \\
R_{K^{*} \rho}^{\mathcal{H}_{i}^{0} \rightarrow D_{(s)}^{*}}= & \frac{\Gamma\left(\mathcal{H}_{i}^{0} \rightarrow D_{s}^{*} K^{*-}\right)}{\Gamma\left(\mathcal{H}_{i}^{0} \rightarrow D^{* 0} \rho^{0}\right)+\Gamma\left(\mathcal{H}_{i}^{0} \rightarrow D^{*+} \rho^{-}\right)}
\end{aligned}
$$

For decaying strange mesons we define

$$
\begin{aligned}
R_{\phi K^{*}}^{\mathcal{H}_{i s} \rightarrow D_{(s)}} & =\frac{\Gamma\left(\mathcal{H}_{i s} \rightarrow D_{s} \phi\right)}{\Gamma\left(\mathcal{H}_{i s} \rightarrow D^{+} K^{* 0}\right)+\Gamma\left(\mathcal{H}_{i s} \rightarrow D^{0} K^{*+}\right)}, \\
R_{\phi K^{*}}^{\mathcal{H}_{i s} \rightarrow D_{(s)}^{*}} & =\frac{\Gamma\left(\mathcal{H}_{i s} \rightarrow D_{s}^{*} \phi\right)}{\Gamma\left(\mathcal{H}_{i s} \rightarrow D^{*+} K^{* 0}\right)+\Gamma\left(\mathcal{H}_{i s} \rightarrow D^{* 0} K^{*+}\right)} .
\end{aligned}
$$

For different final heavy mesons we consider

$$
R_{\rho}^{\mathcal{H}_{i}^{+}}=\frac{\Gamma\left(\mathcal{H}_{i}^{+} \rightarrow D^{*+} \rho^{0}\right)+\Gamma\left(\mathcal{H}_{i}^{+} \rightarrow D^{* 0} \rho^{+}\right)}{\Gamma\left(\mathcal{H}_{i}^{+} \rightarrow D^{+} \rho^{0}\right)+\Gamma\left(\mathcal{H}_{i}^{+} \rightarrow D^{0} \rho^{+}\right)}
$$

$$
\begin{array}{r}
R_{\rho}^{\mathcal{H}_{i}^{0}}=\frac{\Gamma\left(\mathcal{H}_{i}^{0} \rightarrow D^{* 0} \rho^{0}\right)+\Gamma\left(\mathcal{H}_{i}^{0} \rightarrow D^{*+} \rho^{-}\right)}{\Gamma\left(\mathcal{H}_{i}^{0} \rightarrow D^{0} \rho^{0}\right)+\Gamma\left(\mathcal{H}_{i}^{0} \rightarrow D^{+} \rho^{-}\right)}, \\
R_{K^{*}}^{\mathcal{H}_{i s}}=\frac{\Gamma\left(\mathcal{H}_{i s} \rightarrow D^{*+} K^{* 0}\right)+\Gamma\left(\mathcal{H}_{i s} \rightarrow D^{* 0} K^{*+}\right)}{\Gamma\left(\mathcal{H}_{i s} \rightarrow D^{+} K^{* 0}\right)+\Gamma\left(\mathcal{H}_{i s} \rightarrow D^{0} K^{*+}\right)}
\end{array}
$$

The $P V$ and $P^{*} V$ thresholds of neutral nonstrange and of strange charmed mesons are shown in Fig. 1. For charged nonstrange charmed mesons the thresholds are almost coincident with the neutral ones.

\section{A. States in $\tilde{\boldsymbol{H}}$ doublets}

There are candidates of radial excitations of $\left(D_{(s)}, D_{(s)}^{*}\right)$ in the $H$ doublet. In particular, $D_{s 1}^{*}(2700)$ observed by Belle [58] and BABAR [59], with mass and width in Table IV, can be identified with the $n=2$ excitation of $D_{s}^{*}(2112)$. Indeed, the measurement [12]

$\frac{\mathcal{B}\left(D_{s 1}^{*}(2700) \rightarrow D^{*} K\right)}{\mathcal{B}\left(D_{s 1}^{*}(2700) \rightarrow D K\right)}=0.91 \pm 0.13_{\text {stat }} \pm 0.12_{\text {syst }}$,

with $D^{(*)} K=D^{(*) 0} K^{+}+D^{(*)^{+}} K_{S}^{0}$, agrees with the prediction for the first radial excitation of $D_{s}^{*}(2112)$ [60]. The situation is unclear for the states without strangeness. Two resonances can be identified with the members of the $n=2 \tilde{H}$ doublet, $\left(D(2550), D^{*}(2600)\right)$ in Table I, most likely coinciding with $\left(D_{J}^{0}(2580), D_{J}^{* 0}(2650)\right)$ in Table II. However, this classification needs to be further corroborated [16].

The masses of the three states are below the $P^{(*)} V$ thresholds, hence higher radial excitations must be considered for decays into light vector mesons. Using (36)-(39), we compute the ratios $R_{\omega \rho}^{\tilde{D} \rightarrow D}, R_{K^{*} \rho}^{\tilde{D} \rightarrow D_{(s)}}, R_{\omega \rho}^{\tilde{D} \rightarrow D^{*}}, R_{K^{*} \rho}^{\tilde{D} \rightarrow D_{(s)}^{*}}$, 

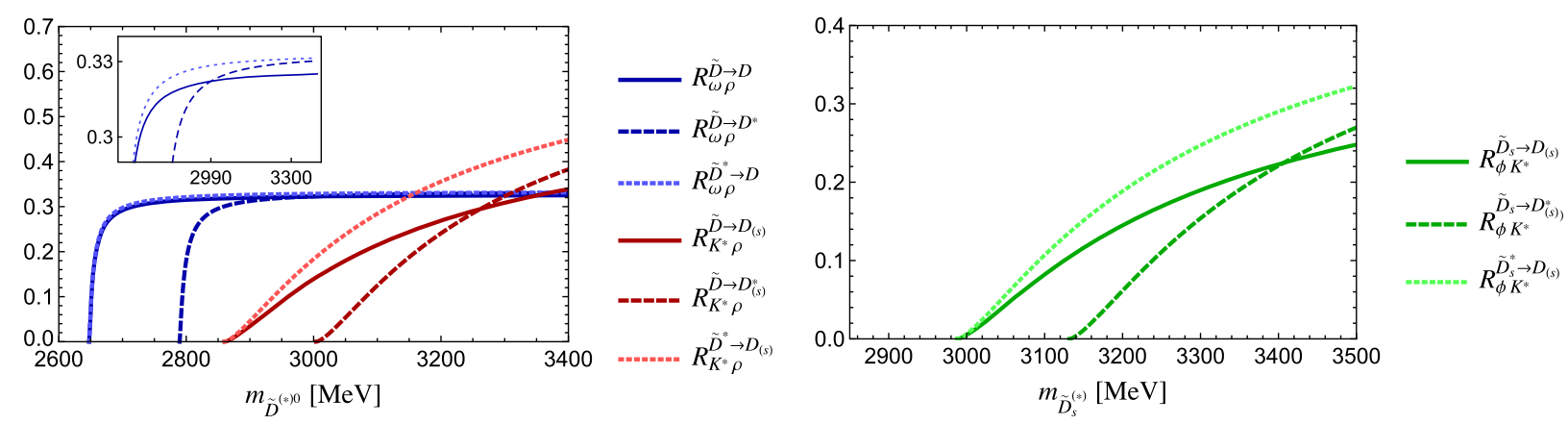

FIG. 2. Ratios in Eqs. (80)-(83) (left) and (84) and (85) (right), evaluated varying the mass of the decaying meson belonging to an excited $(n=3) \tilde{H}$ doublet.

$R_{\omega \rho}^{\tilde{D}^{*} \rightarrow D}$, and $R_{K^{*} \rho}^{\tilde{D}^{*} \rightarrow D_{(s)}}$ in Eqs. (80)-(83) for the $\left(\tilde{D}^{0}, \tilde{D}^{* 0}\right)$ excited doublet. For the strange partners we consider $R_{\phi K^{*}}^{\tilde{D}_{s} \rightarrow D_{(s)}}, R_{\phi K^{*}}^{\tilde{D}_{s} \rightarrow D_{(s)}^{*}}$, and $R_{\phi K^{*}}^{\tilde{D}_{s}^{*} \rightarrow D_{(s)}}$ in Eqs. (84) and (85). In Fig. 2 we depict such observables varying the mass of the decaying meson. We find $R_{\omega \rho}^{\tilde{D} \rightarrow D}>R_{\omega \rho}^{\tilde{D} \rightarrow D^{*}}$ for $m_{\tilde{D}^{0}}>$ $2990 \mathrm{MeV}$, and $R_{K^{*} \rho}^{\tilde{D} \rightarrow D_{(s)}} \simeq R_{K^{*} \rho}^{\tilde{D} \rightarrow D_{(s)}^{*}}$ for $m_{\tilde{D}^{0}} \simeq 3260 \mathrm{MeV}$. The relation (40), varying $m_{\tilde{D}}$ and setting the mass difference between the two spin partners of radial excitations in the range $0 \leq m_{\tilde{D}^{*}}-m_{\tilde{D}} \leq 100 \mathrm{MeV}$, is shown in Fig. 3 for $V=\rho$.

An interesting relation is obtained in terms of the ratios

$$
R_{1}=\frac{\Gamma\left(\tilde{D}^{*} \rightarrow D V\right)}{\Gamma\left(\tilde{D}^{*} \rightarrow D^{*} V\right)}, \quad R_{2}=\frac{\Gamma(\tilde{D} \rightarrow D V)}{\Gamma\left(\tilde{D}^{*} \rightarrow D^{*} V\right)},
$$

using (41):

$$
\begin{aligned}
R_{2}= & \frac{m_{\tilde{D}^{*}}^{4}}{m_{\tilde{D}}^{4}}\left\{\frac{m_{D}}{m_{D^{*}}} \frac{\lambda^{3 / 2}\left(m_{\tilde{D}}^{2}, m_{D}^{2}, m_{V}^{2}\right)}{\lambda^{3 / 2}\left(m_{\tilde{D}^{*}}^{2}, m_{D^{*}}^{2}, m_{V}^{2}\right)}\right. \\
& \left.-2 R_{1} \frac{\lambda^{3 / 2}\left(m_{\tilde{D}}^{2}, m_{D}^{2}, m_{V}^{2}\right)}{\lambda^{3 / 2}\left(m_{\tilde{D}^{*}}^{2}, m_{D}^{2}, m_{V}^{2}\right)}\right\} .
\end{aligned}
$$

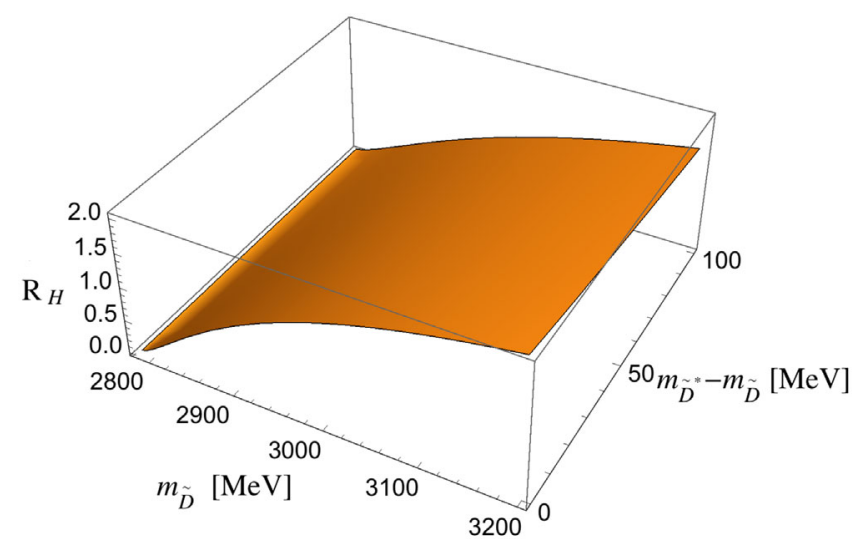

FIG. 3. Ratio (40) for $V=\rho$, varying $m_{\tilde{D}}$ and the mass splitting $m_{\tilde{D}^{*}}-m_{\tilde{D}}$.
This relation is shown for $V=\rho$ in Fig. 4, varying the mass $m_{\tilde{D}}$ of $J^{P}=0^{-}$radial excitation in the range [2900, 3200] $\mathrm{MeV}$, and setting the spin splitting $m_{\tilde{D}^{*}}-m_{\tilde{D}}=$ $40 \pm 20 \mathrm{MeV}$.

\section{B. States in $X$ doublets}

Ratios of decay rates independent of the coupling constant can be written for $\left(D_{1}^{*}, D_{2}\right)$ belonging to the $X$ doublet. They are plotted in Fig. 5 varying the mass of the decaying particle. There are two candidates for the lowestlying $X$ doublet: $D_{J}^{*+}(2760)$ observed in the decay to $D^{0} \pi^{+}$ [6], that is likely to have $J^{P}=1^{-}$, and $D_{s 1}^{*}(2860)$ [11]. Their parameters are in Tables III and IV. Since the $P V$ and not $P^{*} V$ modes are kinematically allowed, we display in Fig. 5 only the ratio $R_{\omega \rho}^{D_{1}^{*} \rightarrow D}$ for $D_{J}^{*+}(2760)$, with the gray vertical line corresponding to the measured $D_{J}^{*+}(2760)$ mass. Identifying $D_{1}^{*}$ with $D_{J}^{*+}(2760)$, we predict

$$
R_{\omega \rho}^{D^{*+}(2760) \rightarrow D}=(29.5 \pm 0.15) \times 10^{-2} .
$$

The ratios $R_{\omega \rho}^{D_{1}^{*} \rightarrow D}$ and $R_{\omega \rho}^{D_{1}^{*} \rightarrow D^{*}}$ are nearly equal for masses larger than $\simeq 3.25 \mathrm{GeV}$, while $R_{K^{*} \rho}^{D_{1}^{*} \rightarrow D_{(s)}}<R_{K^{*} \rho}^{D_{1}^{*} \rightarrow D_{(s)}^{*}}$. The ratios for $D_{s 1}^{*}$ and the spin partner $D_{(s) 2}$ are also in Fig. 5.

The observables in Eqs. (86)-(88), also independent of the coupling constant and involving the same final light vector meson, are displayed in Fig. 6.

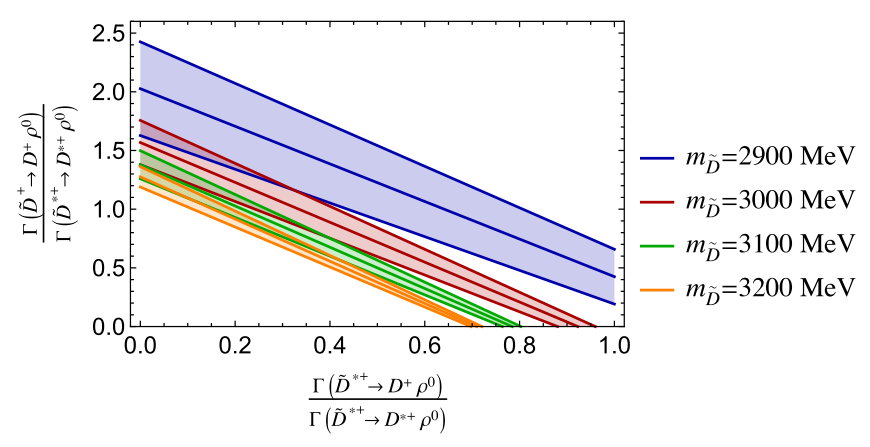

FIG. 4. Relation (91) for several values of $m_{\tilde{D}}$. The bands correspond to the chosen $m_{\tilde{D}^{*}}-m_{\tilde{D}}$ spin splitting. 

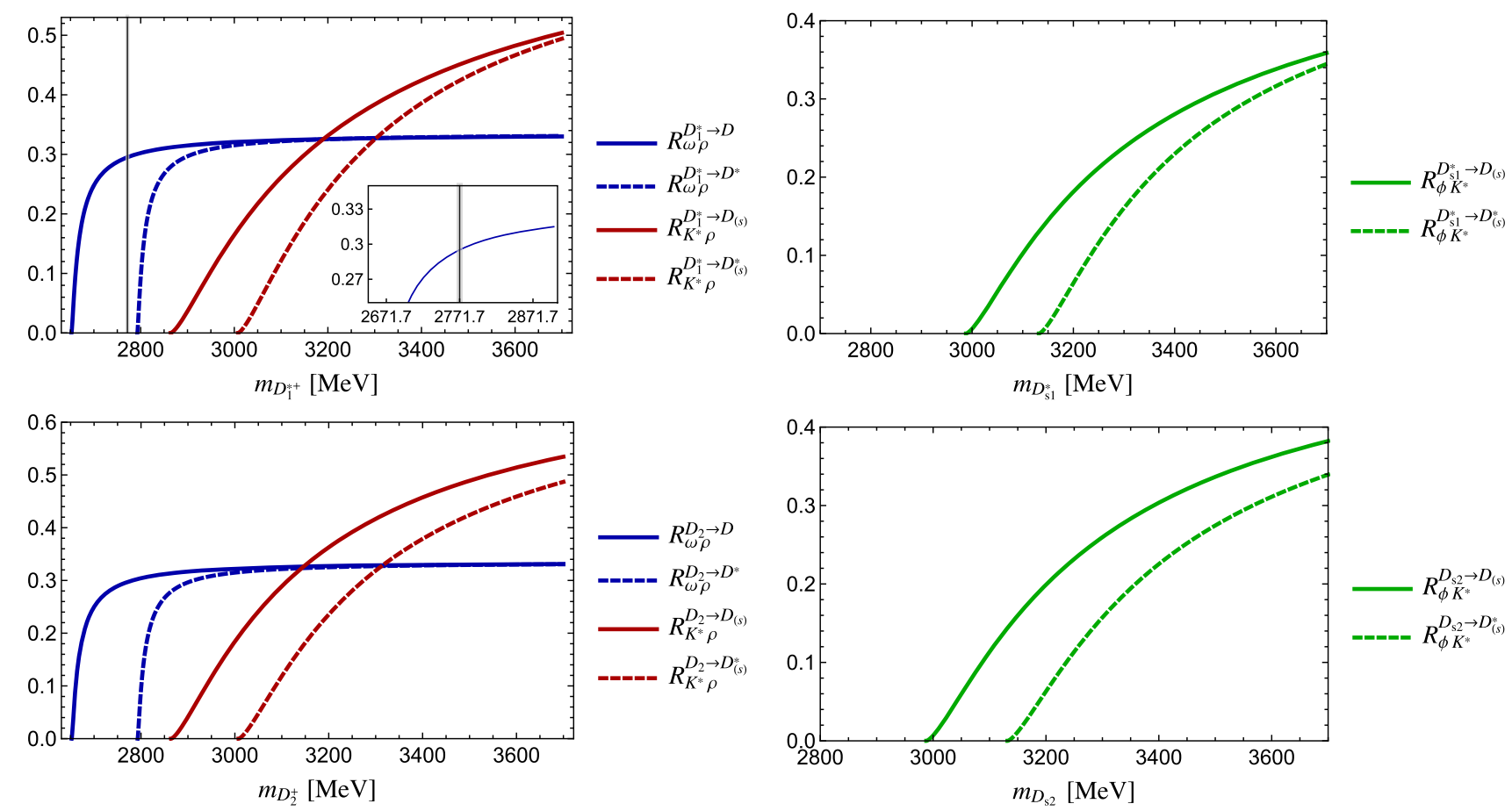

FIG. 5. Ratios in Eqs. (76)-(79) (left) and (84)-(85) (right), evaluated when the decaying particle is $D_{(s) 1}^{*}$ (top) and $D_{(s) 2}$ (bottom) in the $X$ doublet. The gray region corresponds to the measured mass of $D_{J}^{*+}(2760)$, candidate to be identified with $D_{1}^{*}$.

At the chosen order in the effective Lagrangian approach the strong decay widths of the members of the $X$ doublet with a light final pseudoscalar meson depend on the constant $k^{\prime}$ in Eq. (12). Neglecting phase-space suppressed channels (e.g., decays to excited doublets), the widths of the members of the $X$ doublet are determined by the couplings $k^{\prime}$ and $h^{X}$. Saturating the widths of $D^{*+}(2760)$ and $D_{s 1}^{*+}(2860)$ by the modes

$$
\begin{aligned}
D^{*+}(2760) \rightarrow & D^{(*)+} \pi^{0}, D^{(*) 0} \pi^{+}, D^{(*)+} \eta, D_{s}^{(*)} K_{S}, \\
& D^{+} \rho^{0}, D^{0} \rho^{+}, D^{+} \omega \\
D_{s 1}^{*+}(2860) \rightarrow & D^{(*)+} K_{S}, D^{(*) 0} K^{+}, D_{s}^{(*)} \eta, D^{+} K^{* 0}, D^{0} K^{*+},
\end{aligned}
$$

the couplings $k^{\prime}$ and $h^{X}$ can be constrained in the region in Fig. 7, with the bound $\left|k^{\prime}\right|<0.16$.

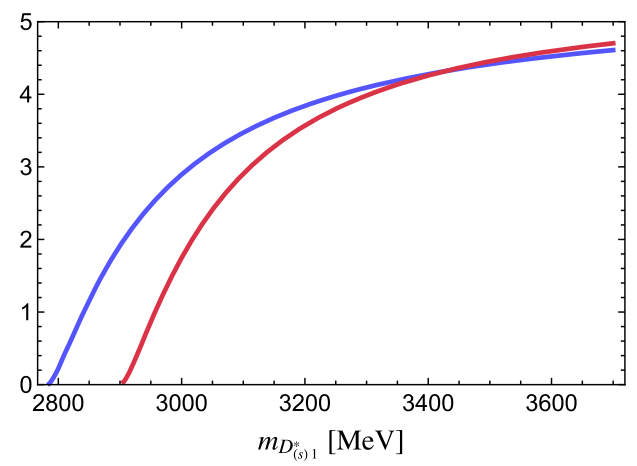

\section{States in $X^{\prime}$ doublet}

In 2006 BABAR observed the $D_{s J}(2860)$ meson decaying to $D K$ [59], which was proposed as the $J^{P}=3^{-}$state in the $c \bar{s} X^{\prime}$ doublet [61]. A subsequent LHCb analysis supported this classification and showed that another state, $D_{s 1}^{*}(2860)$ with $J^{P}=1^{-}$, is present in the same mass region, likely the member of the $X$ doublet [11]. The parameters of the $J^{P}=3^{-}$resonance are in Table IV. LHCb observed another candidate for the $X^{\prime}$ doublet, $D_{3}^{* 0}(2760)$ with parameters in Table III, that can be identified with the nonstrange partner of $D_{s 3}^{*-}(2860)$ [7]. Finally, BABAR and LHCb found a resonance that might be the $J^{P}=2^{-}$state in the $X^{\prime}$ doublet: this is $D^{0}(2750)$ decaying to $D^{*+} \pi^{-}$[5], with parameters in Table I. The LHCb $D^{0}(2740)$ state, observed in $D^{*+} \pi^{-}$[6] (see Table II), likely coincides with it.
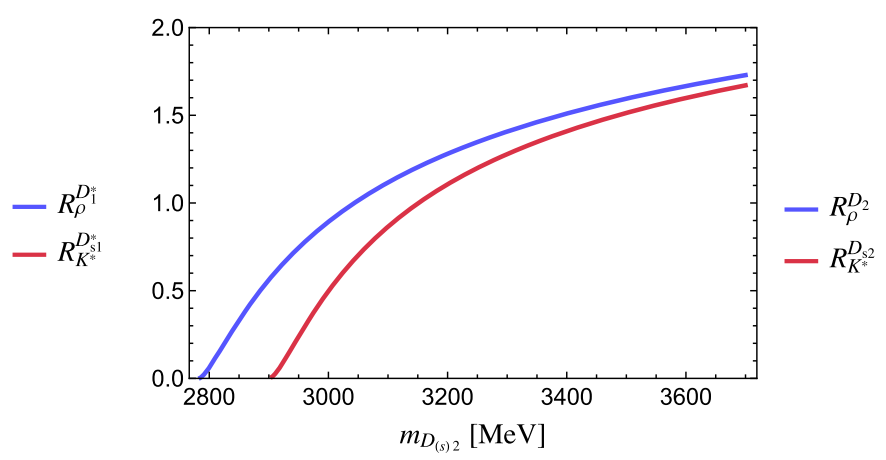

FIG. 6. Ratios (86)-(88) for decaying particle in the $X$ doublet. 


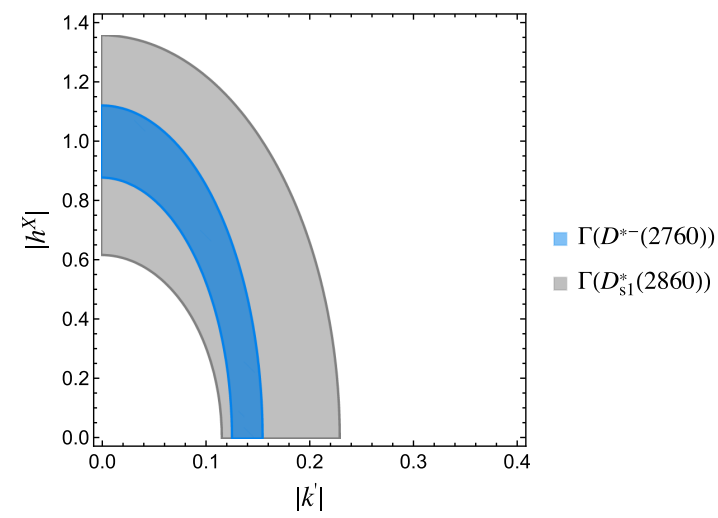

FIG. 7. Bounds for the couplings $k^{\prime}$ and $h^{X}$ from the widths of the $X$ doublet candidates $D^{*+}(2760)$ and $D_{s 1}^{*}(2860)$.

For the two $J^{P}=3^{-}$states, allowed decays to light vector mesons are $D_{3}^{* 0}(2760) \rightarrow D^{+} \rho^{-}, D^{0} \rho^{0}, D^{0} \omega$ and $D_{s 3}^{*+} \rightarrow \bar{D}^{0} K^{*+}, D^{-} K^{* 0}$. We plot in Fig. 8 ratios of widths independent of the coupling constant, varying the mass of the decaying particle. In correspondence to the measured $D_{3}^{* 0}(2760)$ mass we predict

$$
R_{\rho \omega}^{D_{0}^{* 0}(2760) \rightarrow D}=(30.1 \pm 0.2) \times 10^{-2}
$$

and
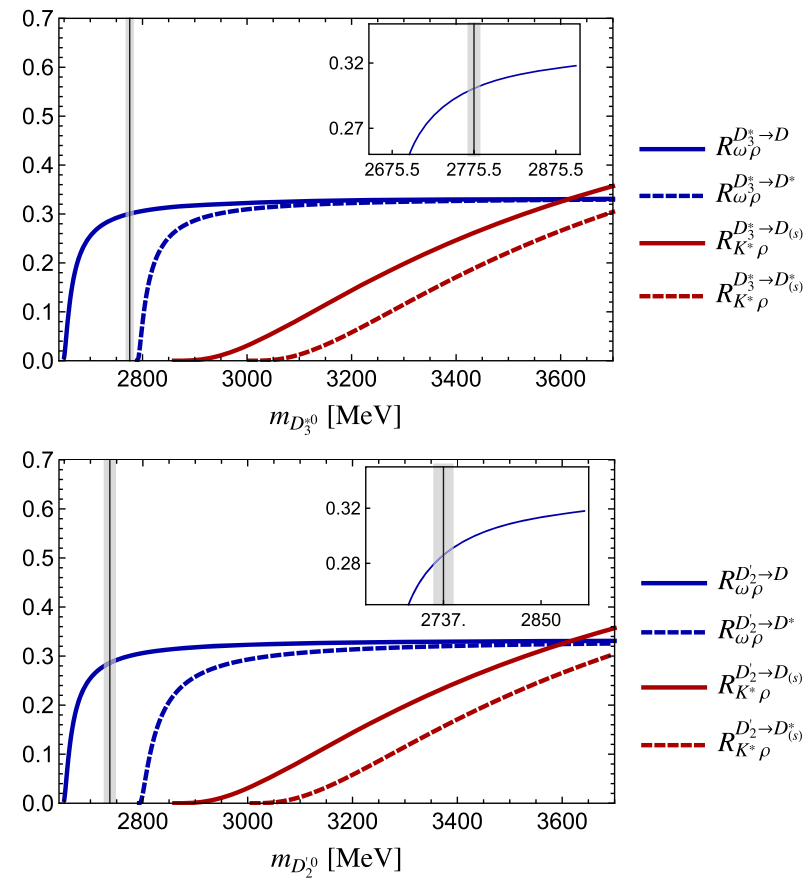

$$
\begin{aligned}
R_{a}^{X^{\prime}} & =\frac{\Gamma\left(D_{3}^{* 0}(2760) \rightarrow D^{+} \rho^{-}\right)+\Gamma\left(D_{3}^{* 0}(2760) \rightarrow D^{0} \rho^{0}\right)}{\Gamma\left(D_{s 3}^{*-}(2860) \rightarrow \bar{D}^{0} K^{*-}\right)+\Gamma\left(D_{s 3}^{*-}(2860) \rightarrow D^{-} K^{* 0}\right)} \\
& =1.6 \pm 0.5, \\
R_{b}^{X^{\prime}} & =\frac{\Gamma\left(D_{3}^{* 0}(2760) \rightarrow D^{0} \omega\right)}{\Gamma\left(D_{s 3}^{*-}(2860) \rightarrow \bar{D}^{0} K^{*-}\right)+\Gamma\left(D_{s 3}^{*-}(2860) \rightarrow D^{-} K^{* 0}\right)} \\
& =0.47 \pm 0.16 .
\end{aligned}
$$

Analogous ratios for the $J^{P}=2^{-}$member of the $X$ doublet, with and without strangeness, are shown in Fig. 8. In the nonstrange case, the candidate is $D^{0}(2740)$. The $D^{+} \rho^{-}$, $D^{0} \rho^{0}, D^{0} \omega$ channels are open, and we predict

$$
R_{\rho \omega}^{D_{2}^{\prime 0}(2740) \rightarrow D}=(28.6 \pm 0.6) \times 10^{-2} .
$$

In the same figure $R_{\rho \omega}^{D_{2}^{\prime} \rightarrow D}$ is plotted versus the mass of $D_{2}^{\prime}$, with the gray region corresponding to the $D^{0}(2740)$ measured mass. Ratios involving the same final light vector meson are displayed in Fig. 9.

In the effective Lagrangian approach, the strong decay widths of the members of the $X^{\prime}$ doublet to a light pseudoscalar meson are controlled by $k=k_{1}+k_{2}$, with $k_{1}$ and $k_{2}$ in Eq. (13). Neglecting phase-space suppressed modes, the widths of the members of the $X^{\prime}$ doublet are determined by the couplings $k$ and $k^{X^{\prime}}$. If $\left(D^{0}(2750)\right.$, $D_{3}^{*}(2760)$ ) and $D_{s 3}^{*}(2860)$ belong to the $X^{\prime}$ doublet, their widths impose constraints on the two constants, as shown in the left panel of Fig. 10 obtained assuming the full widths saturated by
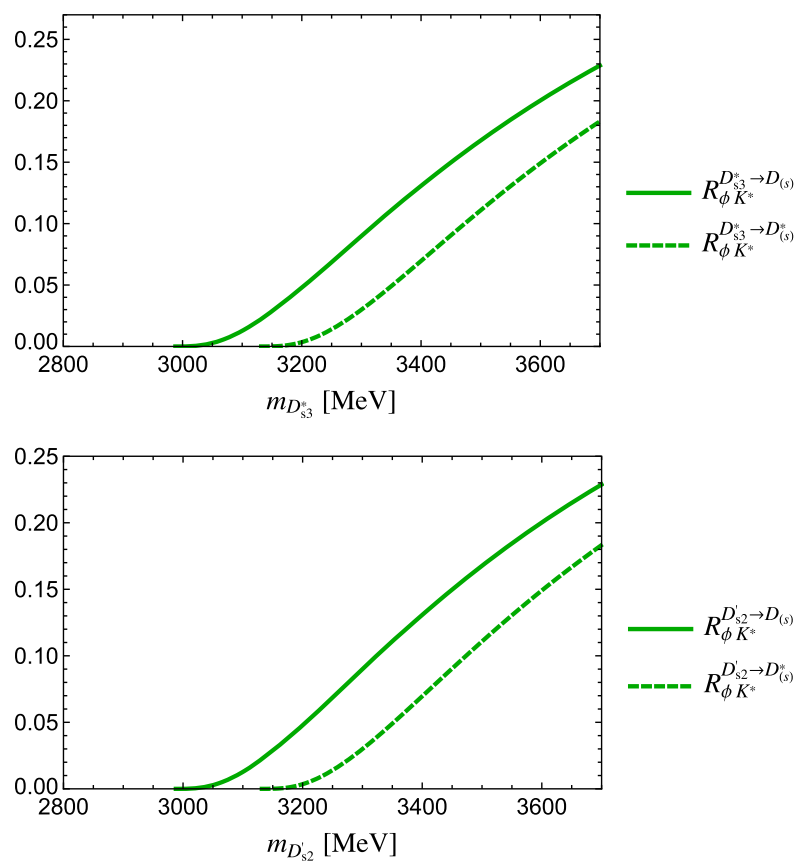

FIG. 8. Ratios in Eqs. (80)-(83) (left) and (84) and (85) (right), evaluated when the decaying particle is $D_{(s) 3}^{*}$ (top) and $D_{(s) 2}^{\prime}$ (bottom) in the $X^{\prime}$ doublet. The gray regions (enlarged in the inset) correspond to the measured mass of $D_{3}^{* 0}(2760)$ candidate as $D_{3}^{*}$, and of $D^{0}(2740)$ candidate for the $D_{2}^{\prime}$ in the $X^{\prime}$ doublet. 

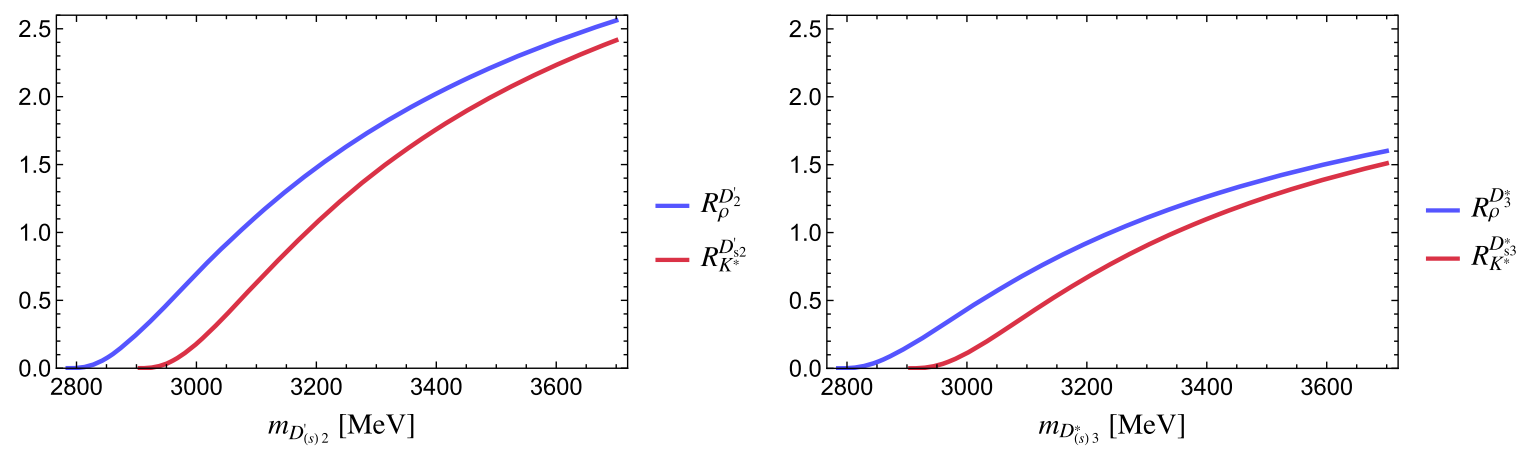

FIG. 9. Ratios (86)-(88) for a decaying particle belonging to $X^{\prime}$.
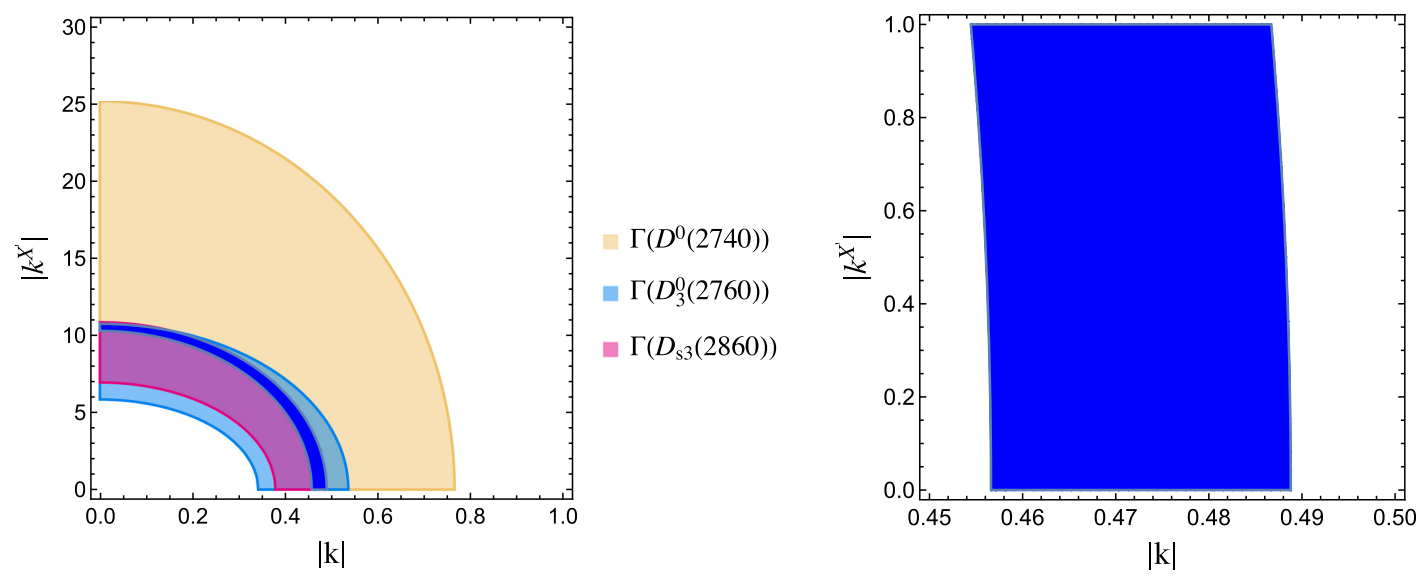

FIG. 10. Left: constraints on the couplings $k, k^{X^{\prime}}$ from the measured widths of $D^{0}(2740)$ [6], $D_{3}^{*}(2760)$ [7], and $D_{s 3}^{*}(2860)$ [11] candidates for the $X^{\prime}$ doublet. In the dark blue region all constraints are fulfilled. Right: coupling region for $\left|k^{X^{\prime}}\right|<1$.

$$
\begin{aligned}
D^{0}(2740) & \rightarrow D^{* 0} \pi^{0}, D^{*+} \pi^{-}, D^{* 0} \eta, D_{s}^{*} K^{-}, D^{+} \rho^{-}, D^{0} \rho^{0}, D^{0} \omega \\
D_{3}^{* 0}(2760) \rightarrow & D^{(*)+} \pi^{-}, D^{(*) 0} \pi^{0}, D^{(*)} \eta, D_{s}^{(*)} K^{-}, \\
& D^{+} \rho^{-}, D^{0} \rho^{0}, D^{0} \omega \\
D_{s 3}^{*}(2860) \rightarrow & \rightarrow D^{(*)+} K_{S}, D^{(*) 0} K^{+}, D_{s}^{(*)} \eta, D^{+} K^{* 0}, D^{0} K^{*+} .
\end{aligned}
$$

For $\left|k^{X^{\prime}}\right|<1$ the coupling region is also shown in Fig. 10 (right panel): $k^{X^{\prime}}$ is unconstrained, while $|k|=0.47 \pm 0.02$, slightly above the value obtained in [16] using the BABAR data $[5,59]$.

\section{States in $\tilde{\boldsymbol{T}}$ doublet}

We analyze the $\tilde{T}$ doublet before $F$ since there is a state that can fit in both of them, and this sequence in the discussion is convenient. For each one of the two states in the $\tilde{T}$ spin doublet we construct ratios of decay rates independent of strong couplings. A $J^{P}=2^{+}$meson has been observed [7], $D_{2}^{*}(3000)$, that could fit in the $\tilde{T}$ or in the $F$ doublet. Hence, we compute the various ratios varying the mass of the decaying particle, then we specialize to the mass of the candidate, as shown in Fig. 11. For $D_{2}^{*}(3000)$ belonging to this doublet we predict

$$
\begin{aligned}
R_{\omega \rho}^{D_{2}^{* 0}(3000) \rightarrow D} & =(33.0 \pm 0.1) \times 10^{-2}, \\
R_{\omega \rho}^{D_{20}^{* 0}(3000) \rightarrow D^{*}} & =(32.6 \pm 0.2) \times 10^{-2}, \\
R_{K_{2}^{*} \rho}^{D_{* 0}(3000) \rightarrow D_{(s)}} & =(23.5 \pm 3.6) \times 10^{-2}, \\
R_{K^{*} \rho}^{D_{* 0}^{* 0}(3000) \rightarrow D_{(s)}^{*}} & =(13.0 \pm 4.5) \times 10^{-2} .
\end{aligned}
$$

Ratios of decay rates involving the same final vector meson are plotted in Fig. 12. For $D_{2}^{*}(3000)$ belonging to $\tilde{T}$ we predict $R_{\rho}^{\tilde{D}_{2}^{*}}=0.22 \pm 0.02$. If the $D_{2}^{*}(3000)$ full width is saturated by the modes $D^{(*) 0} \pi^{0}, D^{(*)+} \pi^{-}, D^{(*) 0} \eta, D_{s}^{(*)} K^{-}$, $D^{(*)+} \rho^{-}, D^{(*) 0} \rho^{0}, D^{(*) 0} \omega, D_{s}^{(*)} K^{*-}$, the two couplings $h^{T}$ in (47) and $\tilde{h}^{\prime}$ in (11) can be constrained to the region in Fig. 13, with the bounds $\left|\tilde{h}^{\prime}\right|<0.135$ and $\left|h^{T}\right|<0.29$.

\section{E. $F$ doublet}

The single ratio independent of the coupling constant in the effective Lagrangian Eq. (75) for $D_{3}$ and for its strange partner $D_{s 3}$ is shown in Fig. 14, obtaining $R_{\omega \rho}^{D_{3} \rightarrow D}<R_{K^{*} \rho}^{D_{3} \rightarrow D_{(s)}}$ for a decaying particle mass below $3.38 \mathrm{GeV}$. 

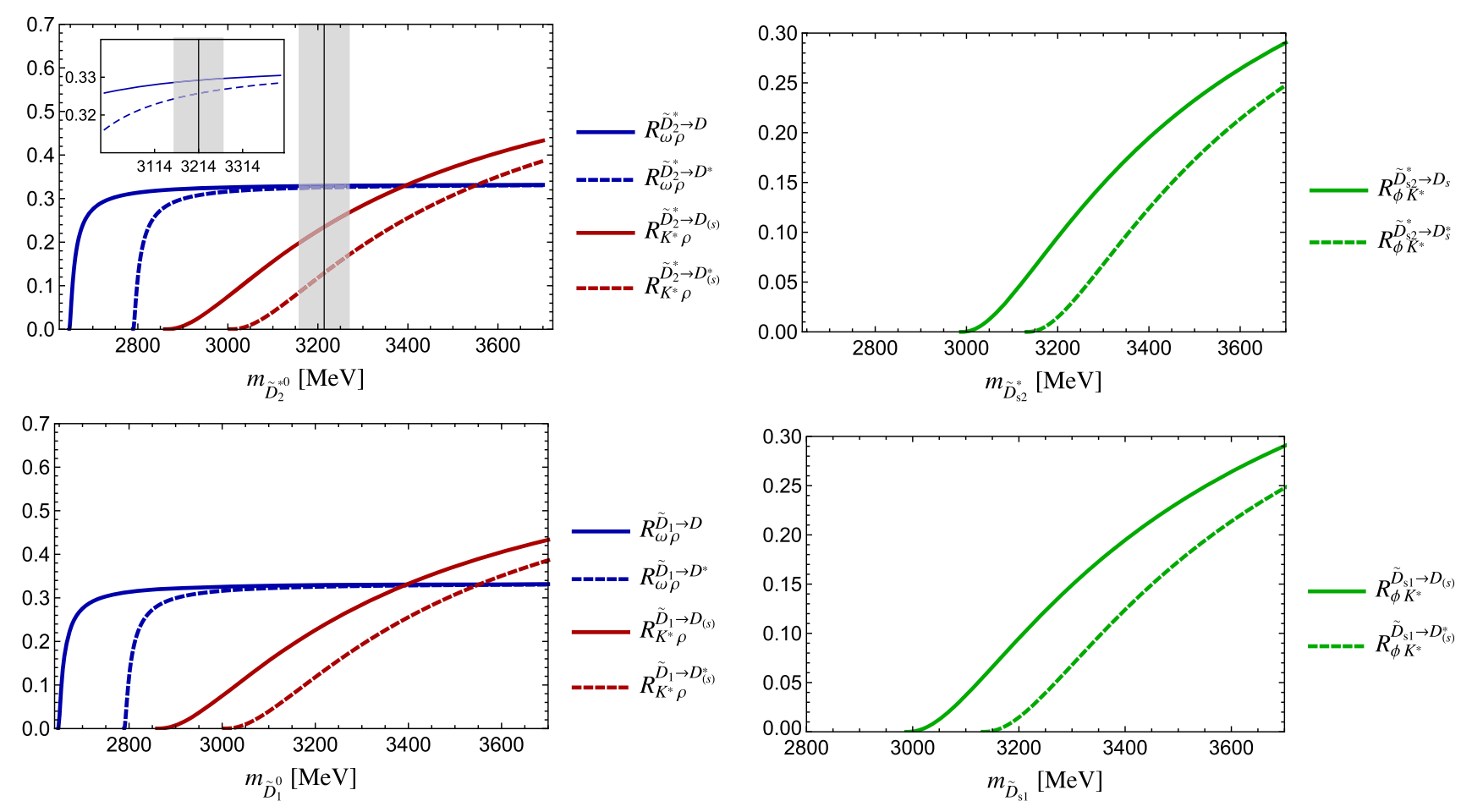

FIG. 11. Ratios in Eqs. (80)-(83) (left) and (84)-(85) (right), evaluated when the decaying particle is $\tilde{D}_{(s) 2}^{*}$ (top) and $\tilde{D}_{(s) 1}$ (bottom) belonging to $\tilde{T}$.
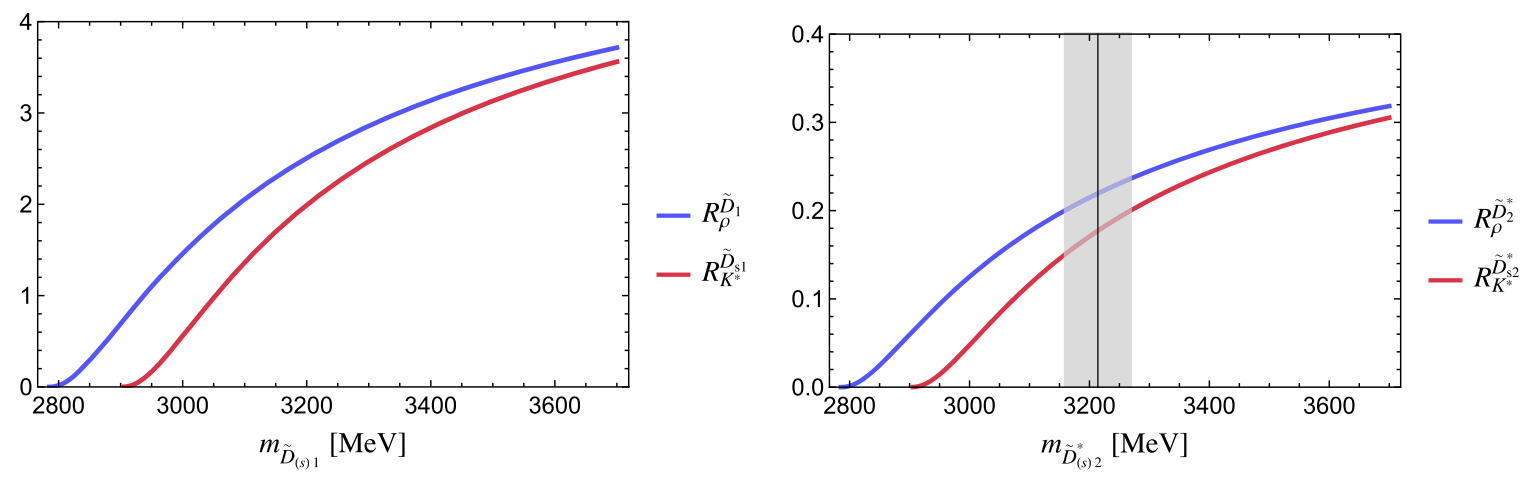

FIG. 12. Ratios (86)-(88) when the decaying particle belongs to the $\tilde{T}$ doublet.

\section{F. More about $D_{2}^{*}(\mathbf{3 0 0 0})$}

The LHCb assignment for this particle is $J^{P}=2^{+}[7]$, and mass and width are compatible with $D_{J}^{* 0}(3000)$ [6] (Tables II and III). It could be identified with the lowest lying $J^{P}=2^{+} n=2$ state $\tilde{D}_{2}^{*}$ in $\tilde{T}$ doublet, or with $D_{2}^{\prime *}$ belonging to the $n=1 F$ doublet. Predictions for the masses of the two states have been worked out in quark models. For example, using the chiral quark model developed in $[62,63]$, the values $m_{\tilde{D}_{2}^{*}}=3035 \mathrm{GeV}$ and $m_{D_{2}^{\prime *}}=$ $3101 \mathrm{GeV}$ have been predicted [64]. As for the identification of $D_{2}^{*}(3000)$, no consensus is reached adopting variants of the quark model. Using a model with instantaneous Bethe-Salpeter potential, identification with $\tilde{D}_{2}^{*}$ is supported [65], while $D_{2}^{*}(3000)$ is preferably interpreted as $D_{2}^{\prime *}$ on the basis of the ${ }^{3} P_{0}$ model for strong decays [66].
The two possibilities lead to different predictions for the $P^{(*)} M$ and to $P^{(*)} V$ widths. Possible transitions to $P^{(*)} M$ are $D_{2}^{* 0}(3000) \rightarrow D^{(*) 0} \pi^{0}, D^{(*)+} \pi^{-}, D^{(*) 0} \eta, D_{s}^{(*)} K^{-}$, leading to the strong coupling independent ratios:

$$
\begin{aligned}
& R_{\pi}^{0}=\frac{\Gamma\left(D_{2}^{* 0}(3000) \rightarrow D^{* 0} \pi^{0}\right)+\Gamma\left(D_{2}^{* 0}(3000) \rightarrow D^{*+} \pi^{-}\right)}{\Gamma\left(D_{2}^{* 0}(3000) \rightarrow D^{0} \pi^{0}\right)+\Gamma\left(D_{2}^{* 0}(3000) \rightarrow D^{+} \pi^{-}\right)}, \\
& R_{\eta}^{0}=\frac{\Gamma\left(D_{2}^{* 0}(3000) \rightarrow D^{0} \eta\right)}{\Gamma\left(D_{2}^{* 0}(3000) \rightarrow D^{0} \pi^{0}\right)+\Gamma\left(D_{2}^{* 0}(3000) \rightarrow D^{+} \pi^{-}\right)},
\end{aligned}
$$




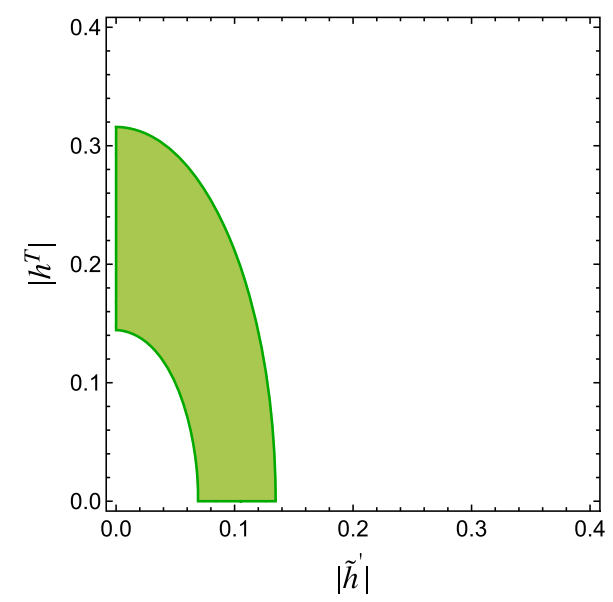

FIG. 13. Bounds on the couplings $\tilde{h}^{\prime}$ in (11) and $h^{T}$ in (47) from the measured width of $D_{2}^{*}(3000)$, assuming that the state belongs to $\tilde{T}$.

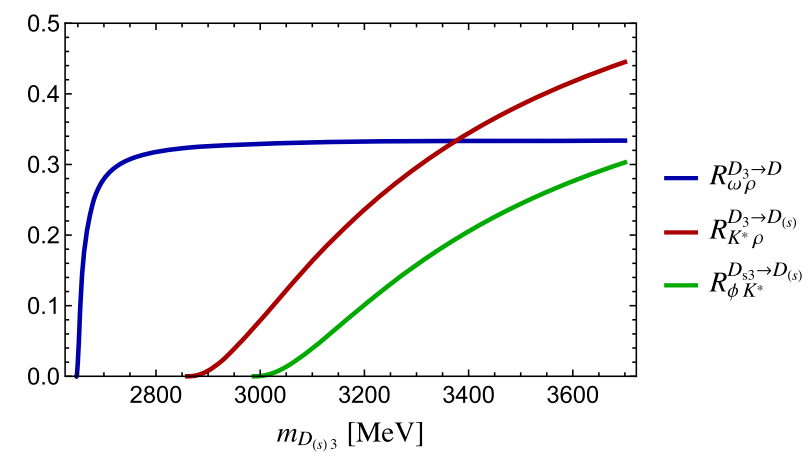

FIG. 14. Ratio (75) for different final states when the decaying particle is $D_{(s) 3}$ in the $F$ doublet.

$$
R_{\eta}^{* 0}=\frac{\Gamma\left(D_{2}^{* 0}(3000) \rightarrow D^{* 0} \eta\right)}{\Gamma\left(D_{2}^{* 0}(3000) \rightarrow D^{0} \pi^{0}\right)+\Gamma\left(D_{2}^{* 0}(3000) \rightarrow D^{+} \pi^{-}\right)},
$$

$$
R_{K}^{0}=\frac{\Gamma\left(D_{2}^{* 0}(3000) \rightarrow D_{s} K^{-}\right)}{\Gamma\left(D_{2}^{* 0}(3000) \rightarrow D^{0} \pi^{0}\right)+\Gamma\left(D_{2}^{* 0}(3000) \rightarrow D^{+} \pi^{-}\right)},
$$

$$
R_{K}^{* 0}=\frac{\Gamma\left(D_{2}^{* 0}(3000) \rightarrow D_{s}^{*} K^{-}\right)}{\Gamma\left(D_{2}^{* 0}(3000) \rightarrow D^{0} \pi^{0}\right)+\Gamma\left(D_{2}^{* 0}(3000) \rightarrow D^{+} \pi^{-}\right)} .
$$

Decay modes of the strange partner of $D_{2}^{*}(3000)$ are $D_{s 2}^{*} \rightarrow D_{s}^{(*)} \eta, D^{(*)+} K_{S}, D^{(*) 0} K^{+}$, with the following ratios:

$$
\begin{gathered}
R_{s, K}^{*}=\frac{\Gamma\left(D_{s 2}^{*+} \rightarrow D^{*+} K_{S}\right)+\Gamma\left(D_{s 2}^{*+} \rightarrow D^{* 0} K^{+}\right)}{\Gamma\left(D_{s 2}^{*+} \rightarrow D^{+} K_{S}\right)+\Gamma\left(D_{s 2}^{*+} \rightarrow D^{0} K^{+}\right)}, \\
R_{s, \eta}=\frac{\Gamma\left(D_{s 2}^{*+} \rightarrow D_{s} \eta\right)}{\Gamma\left(D_{s 2}^{*+} \rightarrow D^{+} K_{S}\right)+\Gamma\left(D_{s 2}^{*+} \rightarrow D^{0} K^{+}\right)}, \\
R_{s, \eta}^{*}=\frac{\Gamma\left(D_{s 2}^{*+} \rightarrow D_{s}^{*} \eta\right)}{\Gamma\left(D_{s 2}^{*+} \rightarrow D^{+} K_{S}\right)+\Gamma\left(D_{s 2}^{*+} \rightarrow D^{0} K^{+}\right)} .
\end{gathered}
$$

The results are different if one identifies $D_{2}^{*}(3000)$ with $\tilde{D}_{2}^{*}$ in the $\tilde{T}$ or with $D_{2}^{* *}$ in the $F$ doublet. We fix the $D_{2}^{* 0}$ mass to the value in Table III with the errors combined in quadrature: $m_{D_{2}^{* 0}}=3214 \pm 57 \mathrm{MeV}$, and for the strange partner we assume $m_{D_{s 2}^{*}}=m_{D_{2}^{* 0}}+100 \mathrm{MeV}$ enlarging the uncertainty: $m_{D_{s 2}^{*}}=3314 \pm 70 \mathrm{MeV}$. The ratios (97)-(101) and (102)-(104) for the two classifications are in Tables VII and VIII. $R_{\pi}^{0}$ and $R_{s, K}^{*}$ have the highest sensitivity to the two classifications.

The two assignments lead to predictions for the spin partner of $D_{2}^{*}(3000)$. For $D_{2}^{*}(3000)$ identified with $\tilde{D}_{2}^{*}$, the spin partner is the $J^{P}=1^{+}$state $\tilde{D}_{1}$, while the spin partner of $D_{2}^{\prime *}$ is $D_{3}$ with $J^{P}=3^{+}$. In the two cases we construct the ratios of decay widths

$$
\begin{gathered}
R_{\mathrm{SP}}^{\tilde{T}}=\frac{\Gamma\left(\tilde{D}_{1}^{0} \rightarrow D^{*+} \pi^{-}\right)+\Gamma\left(\tilde{D}_{1}^{0} \rightarrow D^{* 0} \pi^{0}\right)}{\Gamma\left(\tilde{D}_{2}^{* 0} \rightarrow D^{*+} \pi^{-}\right)+\Gamma\left(\tilde{D}_{2}^{* 0} \rightarrow D^{* 0} \pi^{0}\right)}, \\
R_{\mathrm{SP}}^{F}=\frac{\Gamma\left(D_{3}^{0} \rightarrow D^{*+} \pi^{-}\right)+\Gamma\left(D_{3}^{0} \rightarrow D^{* 0} \pi^{0}\right)}{\Gamma\left(D_{2}^{\prime * 0} \rightarrow D^{*+} \pi^{-}\right)+\Gamma\left(D_{2}^{* 0} \rightarrow D^{* 0} \pi^{0}\right)} .
\end{gathered}
$$

TABLE VII. Ratios in Eqs. (97)-(101) for two different classifications of $D_{2}^{*}(3000)$.

\begin{tabular}{lcccccc}
\hline \hline Doublet & State & \multicolumn{1}{c}{$R_{\pi}^{0}$} & $R_{\eta}^{0}$ & $R_{\eta}^{* 0}$ & $R_{K}^{0}$ & $R_{K}^{* 0}$ \\
\hline$\tilde{T}(n=2)$ & $\tilde{D}_{2}^{* 0}$ & $1.06 \pm 0.03$ & $0.29 \pm 0.01$ & $0.27 \pm 0.02$ & $0.35 \pm 0.020$ & $0.30 \pm 0.03$ \\
$F(n=1)$ & $D_{2}^{* 0}$ & $0.40 \pm 0.015$ & $0.31 \pm 0.01$ & $0.11 \pm 0.01$ & $0.33 \pm 0.02$ & $0.11 \pm 0.01$ \\
\hline \hline
\end{tabular}

TABLE VIII. Ratios defined in Eqs. (102)-(104).

\begin{tabular}{lcccc}
\hline \hline Doublet & State & $R_{s, K}^{*}$ & $R_{s, \eta}$ & $R_{s, \eta}^{*}$ \\
\hline$\tilde{T}(n=2)$ & $\tilde{D}_{s 2}^{*}$ & $1.02 \pm 0.04$ & $0.31 \pm 0.01$ & $0.29 \pm 0.03$ \\
$F(n=1)$ & $D_{s 2}^{\prime *}$ & $0.40 \pm 0.02$ & $0.28 \pm 0.01$ & $0.10 \pm 0.01$ \\
\hline \hline
\end{tabular}



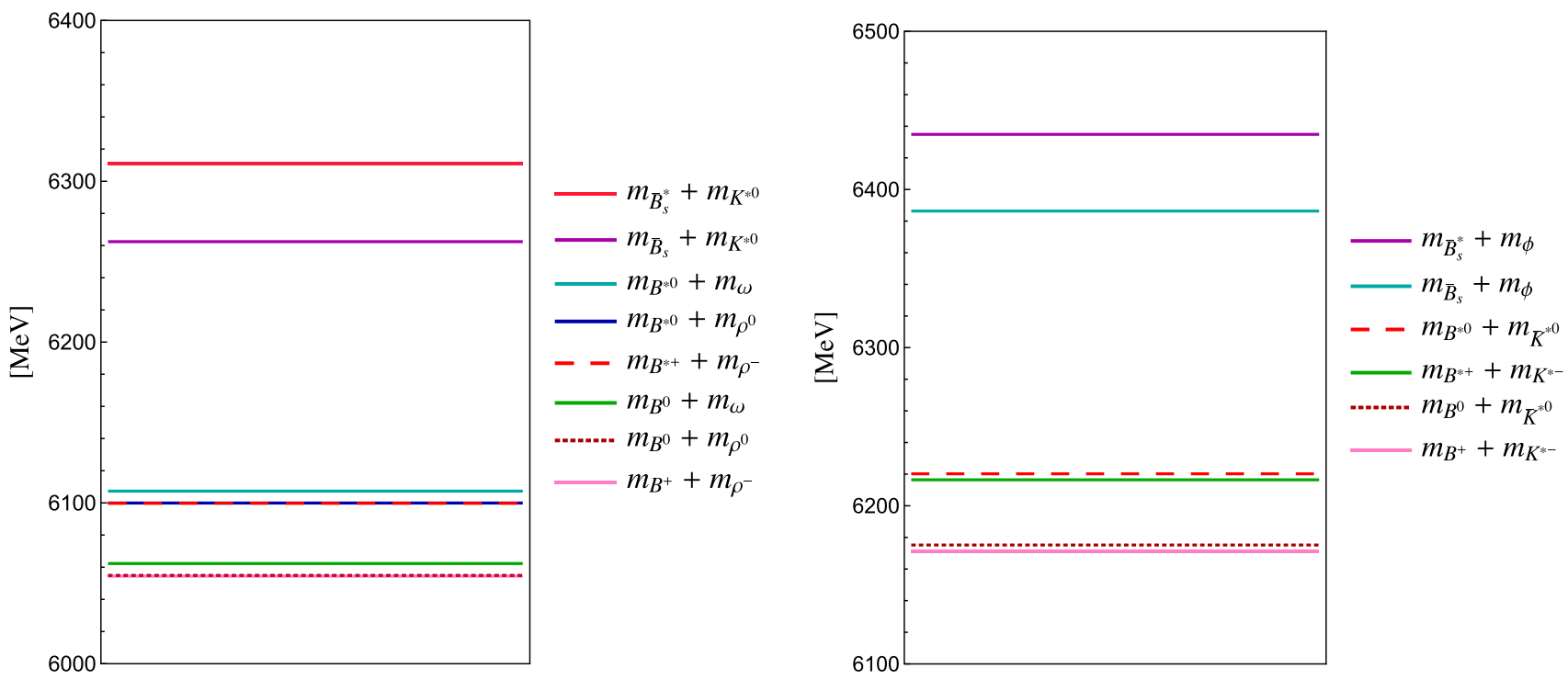

FIG. 15. $\quad P V$ and $P^{*} V$ thresholds for neutral nonstrange (left) and strange beauty mesons (right).

Varying conservatively the mass of $\tilde{D}_{1}$ in the range $\left[m_{D_{2}^{*}(3000)}-100 \mathrm{MeV}, m_{D_{2}^{*}(3000)}\right]$ and the mass of $D_{3}$ in $\left[m_{D_{2}^{*}(3000)}, m_{D_{2}^{*}(3000)}+100 \mathrm{MeV}\right]$ we obtain

$$
1.2 \leq R_{\mathrm{SP}}^{\tilde{T}} \leq 1.7, \quad 1.7 \leq R_{\mathrm{SP}}^{\tilde{T}} \leq 2.6
$$

\section{NUMERICAL ANALYSIS: BEAUTY}

The flavor symmetry allows one to extend the analysis to the beauty sector. The $\mathcal{H}_{i} \rightarrow P^{(*)} V$ thresholds, for $\mathcal{H}_{i}$ a neutral beauty or a beauty-strange meson, are displayed in Fig. 15. No one of the observed excited beauty mesons are above the $P^{(*)} V$ thresholds; therefore, our predictions hold for higher excitations. We define ratios of decay widths for charged and for neutral nonstrange decaying beauty mesons as

$$
\begin{aligned}
R_{\omega \rho}^{\mathcal{H}_{i}^{+} \rightarrow B} & =\frac{\Gamma\left(\mathcal{H}_{i}^{+} \rightarrow B^{+} \omega\right)}{\Gamma\left(\mathcal{H}_{i}^{+} \rightarrow B^{+} \rho^{0}\right)+\Gamma\left(\mathcal{H}_{i}^{+} \rightarrow B^{0} \rho^{+}\right)}, \\
R_{K_{i}^{*} \rho}^{\mathcal{H}_{+}^{+} \rightarrow B_{(s)}} & =\frac{\Gamma\left(\mathcal{H}_{i}^{+} \rightarrow \bar{B}_{s} K^{*+}\right)}{\Gamma\left(\mathcal{H}_{i}^{+} \rightarrow B^{+} \rho^{0}\right)+\Gamma\left(\mathcal{H}_{i}^{+} \rightarrow B^{0} \rho^{+}\right)}, \\
R_{\omega \rho}^{\mathcal{H}_{i}^{+} \rightarrow B^{*}} & =\frac{\Gamma\left(\mathcal{H}_{i}^{+} \rightarrow B^{*+} \omega\right)}{\Gamma\left(\mathcal{H}_{i}^{+} \rightarrow B^{*+} \rho^{0}\right)+\Gamma\left(\mathcal{H}_{i}^{+} \rightarrow B^{* 0} \rho^{+}\right)}, \\
R_{K^{*} \rho}^{\mathcal{H}_{i}^{+} \rightarrow B_{(s)}^{*}} & =\frac{\Gamma\left(\mathcal{H}_{i}^{+} \rightarrow \bar{B}_{s}^{*} K^{*+}\right)}{\Gamma\left(\mathcal{H}_{i}^{+} \rightarrow B^{*+} \rho^{0}\right)+\Gamma\left(\mathcal{H}_{i}^{+} \rightarrow B^{* 0} \rho^{+}\right)},
\end{aligned}
$$

and

$$
R_{\omega \rho}^{\mathcal{H}_{i}^{0} \rightarrow B}=\frac{\Gamma\left(\mathcal{H}_{i}^{0} \rightarrow B^{0} \omega\right)}{\Gamma\left(\mathcal{H}_{i}^{0} \rightarrow B^{0} \rho^{0}\right)+\Gamma\left(\mathcal{H}_{i}^{0} \rightarrow B^{+} \rho^{-}\right)},
$$

$$
\begin{aligned}
R_{K^{*} \rho}^{\mathcal{H}_{i}^{0} \rightarrow B_{(s)}} & =\frac{\Gamma\left(\mathcal{H}_{i}^{0} \rightarrow \bar{B}_{s} K^{* 0}\right)}{\Gamma\left(\mathcal{H}_{i}^{0} \rightarrow B^{0} \rho^{0}\right)+\Gamma\left(\mathcal{H}_{i}^{0} \rightarrow B^{+} \rho^{-}\right)}, \\
R_{\omega \rho}^{\mathcal{H}_{i}^{0} \rightarrow B^{*}} & =\frac{\Gamma\left(\mathcal{H}_{i}^{0} \rightarrow B^{* 0} \omega\right)}{\Gamma\left(\mathcal{H}_{i}^{0} \rightarrow B^{* 0} \rho^{0}\right)+\Gamma\left(\mathcal{H}_{i}^{0} \rightarrow B^{*+} \rho^{-}\right)},
\end{aligned}
$$

$R_{K^{*} \rho}^{\mathcal{H}_{i}^{0} \rightarrow B_{(s)}^{*}}=\frac{\Gamma\left(\mathcal{H}_{i}^{0} \rightarrow \bar{B}_{s}^{*} K^{* 0}\right)}{\Gamma\left(\mathcal{H}_{i}^{0} \rightarrow B^{* 0} \rho^{0}\right)+\Gamma\left(\mathcal{H}_{i}^{0} \rightarrow B^{*+} \rho^{-}\right)}$.

Ratios of decay widths can also be constructed for beauty mesons with strangeness:

$R_{\phi K^{*}}^{\mathcal{H}_{i s} \rightarrow B_{(s)}}=\frac{\Gamma\left(\mathcal{H}_{i s} \rightarrow \bar{B}_{s} \phi\right)}{\Gamma\left(\mathcal{H}_{i s} \rightarrow B^{0} \bar{K}^{* 0}\right)+\Gamma\left(\mathcal{H}_{i s} \rightarrow B^{+} K^{*-}\right)}$,

$R_{\phi K^{*}}^{\mathcal{H}_{i s} \rightarrow B_{(s)}^{*}}=\frac{\Gamma\left(\mathcal{H}_{i s} \rightarrow \bar{B}_{s}^{*} \phi\right)}{\Gamma\left(\mathcal{H}_{i s} \rightarrow B^{*+} \bar{K}^{* 0}\right)+\Gamma\left(\mathcal{H}_{i s} \rightarrow B^{*+} K^{*-}\right)}$.

Ratios of decay widths with the same final $V$ meson are also independent of strong couplings:

$$
R_{\rho}^{\mathcal{H}_{i}^{+}}=\frac{\Gamma\left(\mathcal{H}_{i}^{+} \rightarrow B^{*+} \rho^{0}\right)+\Gamma\left(\mathcal{H}_{i}^{+} \rightarrow B^{* 0} \rho^{+}\right)}{\Gamma\left(\mathcal{H}_{i}^{+} \rightarrow B^{+} \rho^{0}\right)+\Gamma\left(\mathcal{H}_{i}^{+} \rightarrow B^{0} \rho^{+}\right)}
$$

$$
R_{\rho}^{\mathcal{H}_{i}^{0}}=\frac{\Gamma\left(\mathcal{H}_{i}^{0} \rightarrow B^{* 0} \rho^{0}\right)+\Gamma\left(\mathcal{H}_{i}^{0} \rightarrow B^{*+} \rho^{-}\right)}{\Gamma\left(\mathcal{H}_{i}^{0} \rightarrow B^{0} \rho^{0}\right)+\Gamma\left(\mathcal{H}_{i}^{0} \rightarrow B^{+} \rho^{-}\right)},
$$



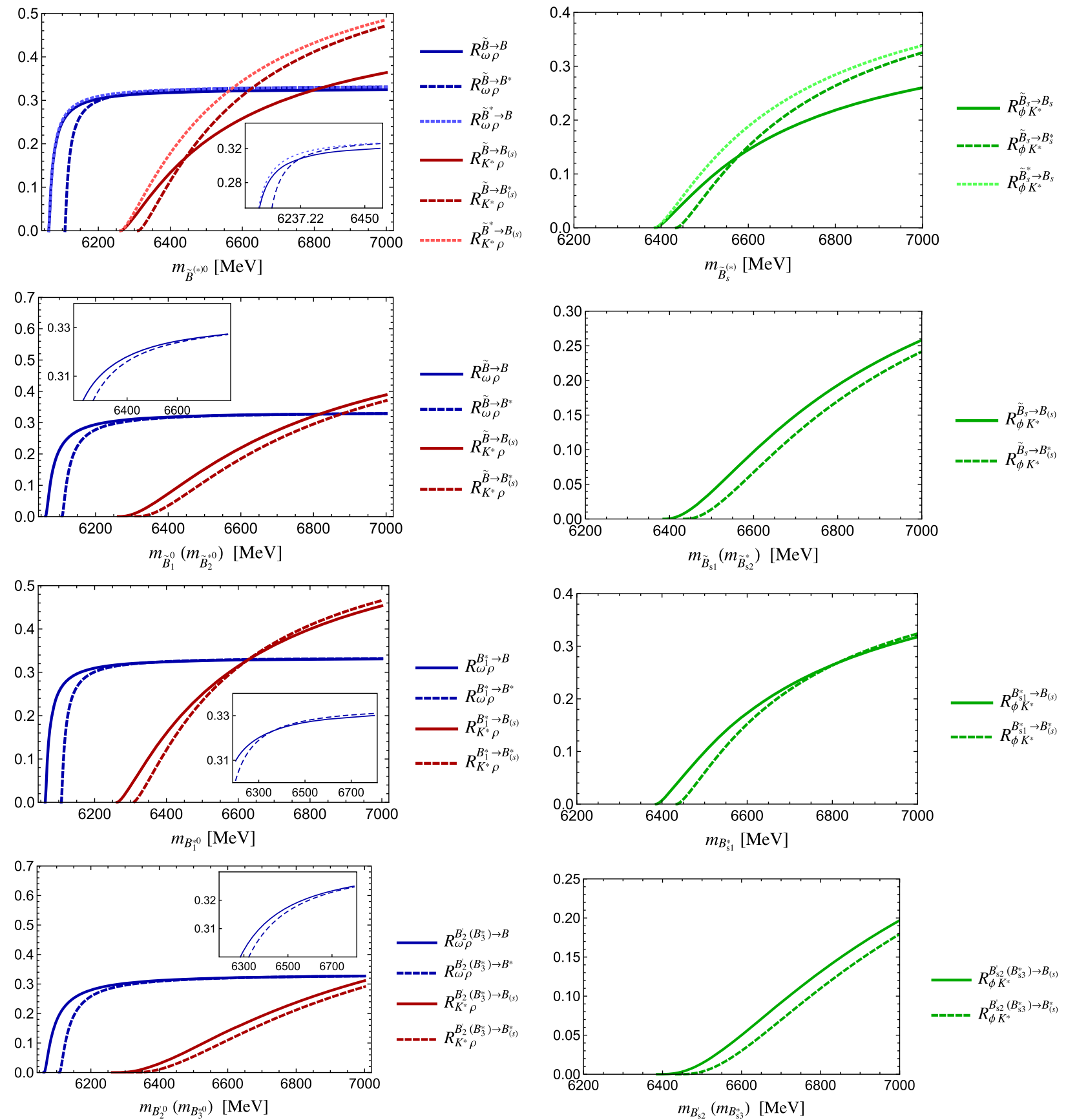

FIG. 16. Ratios (112)-(115) (left) and (116) and (117) (right) for decaying particles belonging to the $b \bar{q}(b \bar{s})$ doublet $\tilde{H}$ (top row), $\tilde{T}$ (second row), $\tilde{X}$ (third row), and $\tilde{X}^{\prime}$ (bottom row).

$R_{K^{*}}^{\mathcal{H}_{i s}}=\frac{\Gamma\left(\mathcal{H}_{i s} \rightarrow B^{*+} K^{*-}\right)+\Gamma\left(\mathcal{H}_{i s} \rightarrow B^{* 0} \bar{K}^{* 0}\right)}{\Gamma\left(\mathcal{H}_{i s} \rightarrow B^{+} K^{*-}\right)+\Gamma\left(\mathcal{H}_{i s} \rightarrow B^{0} \bar{K}^{* 0}\right)}$

\section{A. $H$ doublet}

The ratios (112)-(115) and (116) and (117) evaluated when the decaying particle in $\tilde{H}$, are in Fig. 16. For $m_{\tilde{B}^{0}}<$ $6237.22 \mathrm{MeV}$ one has $R_{\omega \rho}^{\tilde{B} \rightarrow B}>R_{\omega \rho}^{\tilde{B} \rightarrow B^{*}}$. In $\tilde{B}_{s}$ decays, for $m_{\tilde{B}_{s}}>6576.8 \mathrm{MeV}$ one predicts $R_{\phi K^{*}}^{\tilde{B}_{s} \rightarrow B_{s}}<R_{\phi K^{*}}^{\tilde{B}_{s} \rightarrow B_{s}^{*}}$. Other ratios show similar features, namely $R_{K^{*} \rho}^{\tilde{B} \rightarrow B_{(s)}}<R_{K^{*} \rho}^{\tilde{B} \rightarrow B_{(s)}^{*}}$ for $m_{\tilde{B}^{0}}>6441.3 \mathrm{MeV}$.

\section{B. $\tilde{T}$ doublet}

Presenting the results in Fig. 16 we do not distinguish the decaying $\tilde{B}_{1}$ or $\tilde{B}_{2}^{*}$, which have the same expressions for the ratios. The observables in (118) and (120) are displayed in Fig. 17. 

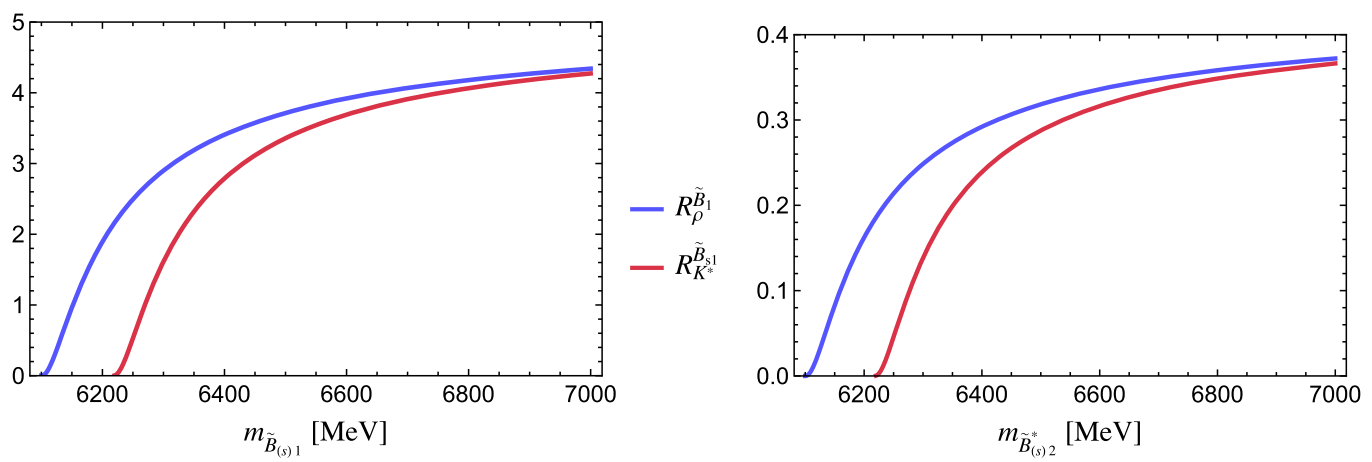

$$
\begin{aligned}
& -R_{\rho_{2}^{*}}^{\tilde{B}_{2}^{*}} \\
& -R_{K^{2}}^{\tilde{B}_{2}^{*}}
\end{aligned}
$$
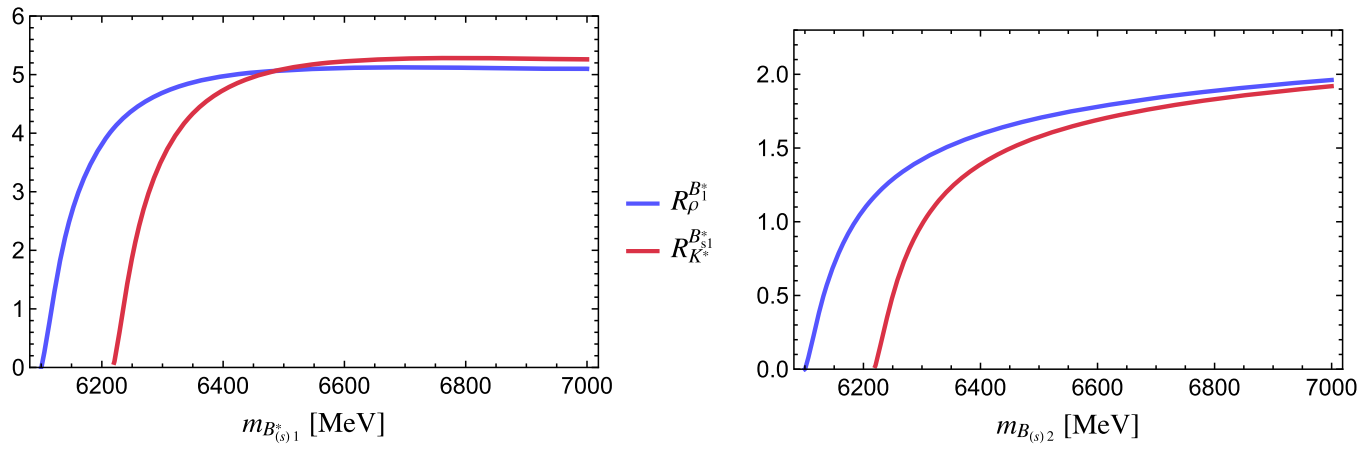

$$
\begin{aligned}
& -R_{\rho_{2}}^{B_{2}} \\
& -R_{K^{2}}^{B_{2}}
\end{aligned}
$$
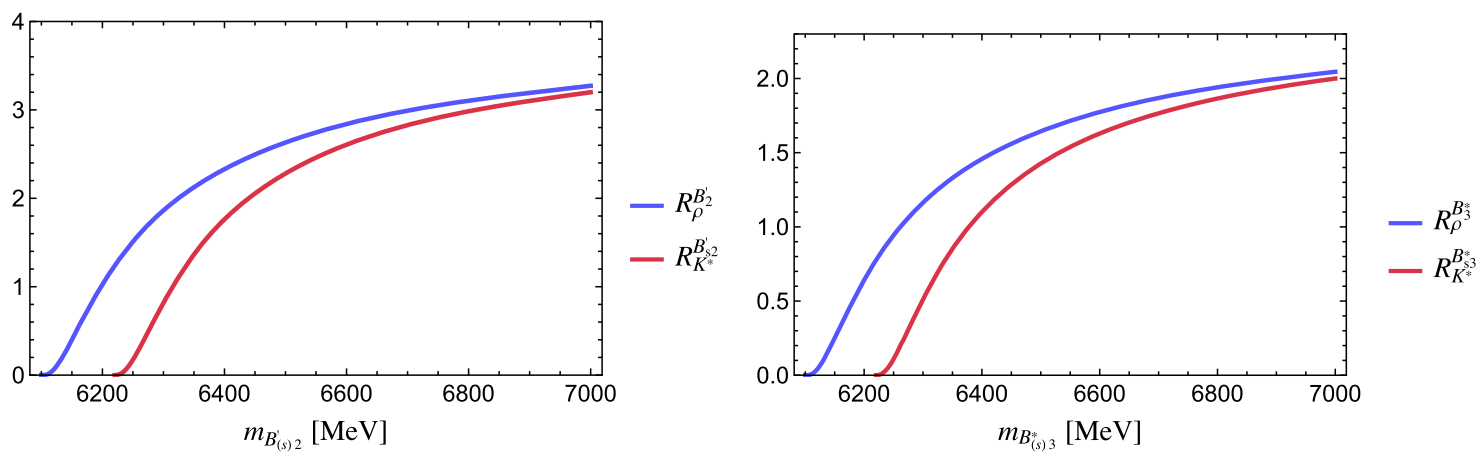

FIG. 17. Ratios (118) and (120) for decaying particles in the $b \bar{q}(b \bar{s}) \tilde{T}$ doublet (top row), $\tilde{X}$ (middle row), and $\tilde{X}^{\prime}$ (bottom row).

\section{C. $X$ doublet}

Ratios of decay rates for beauty mesons in the $X$ doublet are in Fig. 16. When the decaying particle is $B_{1}^{*}$, the two ratios $R_{\omega \rho}^{B_{1}^{*} \rightarrow B}$ and $R_{\omega \rho}^{B_{1}^{*} \rightarrow B^{*}}$ become almost coincident for

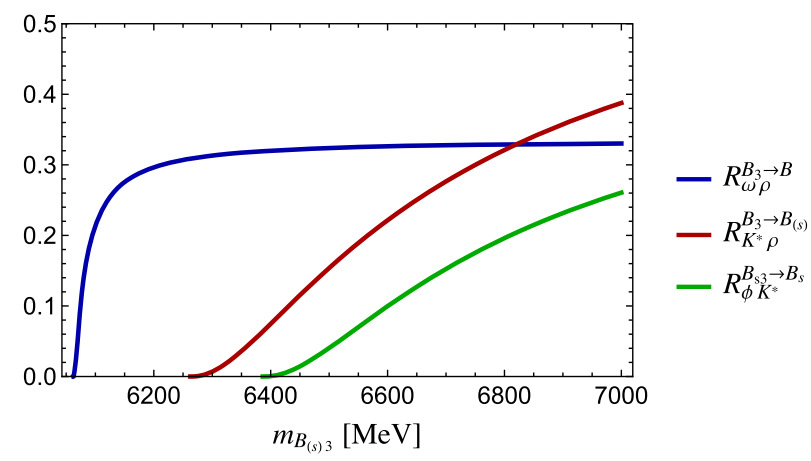

FIG. 18. Ratio (75) for several final states, for a decaying $B_{(s) 3}$ in the $F$ doublet.
$m_{B_{1}^{* 0}} \simeq 6367 \mathrm{MeV}$. Ratios involving the same final light vector meson are in Fig. 17.

\section{D. $X^{\prime}$ doublet}

The considered ratios have the same expressions for the two members in the $X^{\prime}$ doublet. Those defined in Eqs. (112)-(115) and (116) and (117) are displayed in Fig. 16, those with the same final vector meson in Fig. 17.

\section{E. $F$ doublet}

For this doublet there is only one ratio independent of the coupling constants, the one in Eq. (75) for spin 3 meson. The results displayed in Fig. 18 show the hierarchy $R_{\omega \rho}^{B_{3} \rightarrow B}>R_{K^{*} \rho}^{B_{3} \rightarrow B_{(s)}}$ for $m_{B_{3}}<3375 \mathrm{MeV}$.

\section{CONCLUSIONS}

The construction of a QCD-based framework to classify the excited resonances with open charm and beauty and to 
describe their decays is needed in view of the ongoing and forthcoming experimental investigations. Since orbital and radial excitations can be above the thresholds for decays to light vector mesons, we have worked out effective Lagrangian terms governing the strong transition of a heavy meson to a light vector meson and a member of the lowest-lying heavy-light spin doublet, in the HQ limit. We have defined observables independent of the couplings in the Lagrangian, and made predictions varying the mass of the decaying particle. The HQ limit is considered the guideline for the description in the actual cases. Our methods can be exploited for a few observed states with uncertain identification, namely $D_{2}^{*}(3000)$ for which we have compared predictions corresponding to two different classifications. Among the various tasks left to new analyses there are the computation of the various strong couplings and the classification of the subleading Lagrangian terms, which is particularly interesting in case of charm.

\section{ACKNOWLEDGMENTS}

We thank A. Palano for discussions. Work was carried out within the INFN Project QFT-HEP.
[1] H.-X. Chen, W. Chen, X. Liu, Y.-R. Liu, and S.-L. Zhu, Rep. Prog. Phys. 80, 076201 (2017).

[2] C. Patrignani et al. (Particle Data Group), Chin. Phys. C 40, 100001 (2016).

[3] R. Aaij et al. (LHCb Collaboration), Phys. Rev. Lett. 118, 182001 (2017).

[4] R. Aaij et al. (LHCb Collaboration), Phys. Rev. Lett. 119, 112001 (2017).

[5] P. del Amo Sanchez et al. (BABAR Collaboration), Phys. Rev. D 82, 111101 (2010).

[6] R. Aaij et al. (LHCb Collaboration), J. High Energy Phys. 09 (2013) 145.

[7] R. Aaij et al. (LHCb Collaboration), Phys. Rev. D 94, 072001 (2016).

[8] R. Aaij et al. (LHCb Collaboration), Phys. Rev. D 92, 032002 (2015).

[9] R. Aaij et al. (LHCb Collaboration), J. High Energy Phys. 10 (2012) 151.

[10] J. P. Lees et al. (BABAR Collaboration), Phys. Rev. D 91, 052002 (2015).

[11] R. Aaij et al. (LHCb Collaboration), Phys. Rev. Lett. 113, 162001 (2014).

[12] B. Aubert et al. (BABAR Collaboration), Phys. Rev. D 80, 092003 (2009).

[13] T. A. Aaltonen et al. (CDF Collaboration), Phys. Rev. D 90, 012013 (2014).

[14] R. Aaij et al. (LHCb Collaboration), J. High Energy Phys. 04 (2015) 024.

[15] A. M. Sirunyan et al. (CMS Collaboration), Eur. Phys. J. C 78, 939 (2018).

[16] P. Colangelo, F. De Fazio, F. Giannuzzi, and S. Nicotri, Phys. Rev. D 86, 054024 (2012).

[17] Z.-G. Wang, Commun. Theor. Phys. 66, 671 (2016).

[18] P. Gupta and A. Upadhyay, Phys. Rev. D 97, 014015 (2018).

[19] R. Casalbuoni, A. Deandrea, N. Di Bartolomeo, R. Gatto, F. Feruglio, and G. Nardulli, Phys. Lett. B 292, 371 (1992).

[20] R. Casalbuoni, A. Deandrea, N. Di Bartolomeo, R. Gatto, F. Feruglio, and G. Nardulli, Phys. Rep. 281, 145 (1997).

[21] M. Neubert, Phys. Rep. 245, 259 (1994).

[22] A. F. Falk, Nucl. Phys. B378, 79 (1992).

[23] M. B. Wise, Phys. Rev. D 45, R2188 (1992).
[24] G. Burdman and J. F. Donoghue, Phys. Lett. B 280, 287 (1992).

[25] P. L. Cho, Phys. Lett. B 285, 145 (1992).

[26] T.-M. Yan, H.-Y. Cheng, C.-Y. Cheung, G.-L. Lin, Y. C. Lin, and H.-L. Yu, Phys. Rev. D 46, 1148 (1992); 55, 5851(E) (1997).

[27] R. Casalbuoni, A. Deandrea, N. Di Bartolomeo, R. Gatto, F. Feruglio, and G. Nardulli, Phys. Lett. B 299, 139 (1993).

[28] P. Colangelo, F. De Fazio, and G. Nardulli, Phys. Lett. B 334, 175 (1994).

[29] P. Colangelo, G. Nardulli, A. Deandrea, N. Di Bartolomeo, R. Gatto, and F. Feruglio, Phys. Lett. B 339, 151 (1994).

[30] V. M. Belyaev, V. M. Braun, A. Khodjamirian, and R. Ruckl, Phys. Rev. D 51, 6177 (1995).

[31] P. Colangelo, F. De Fazio, G. Nardulli, N. Di Bartolomeo, and R. Gatto, Phys. Rev. D 52, 6422 (1995).

[32] P. Colangelo and F. De Fazio, Eur. Phys. J. C 4, 503 (1998).

[33] D. Becirevic, B. Blossier, E. Chang, and B. Haas, Phys. Lett. B 679, 231 (2009).

[34] D. Becirevic, E. Chang, and A. Le Yaouanc, arXiv: 1203.0167.

[35] M. Bando, T. Kugo, and K. Yamawaki, Nucl. Phys. B259, 493 (1985).

[36] M. Bando, T. Kugo, and K. Yamawaki, Phys. Rep. 164, 217 (1988).

[37] H. Georgi, Phys. Rev. Lett. 63, 1917 (1989).

[38] H. Georgi, Nucl. Phys. B331, 311 (1990).

[39] P. Ko, Phys. Rev. D 47, 1964 (1993).

[40] J. Schechter and A. Subbaraman, Phys. Rev. D 48, 332 (1993).

[41] N. Kitazawa and T. Kurimoto, Phys. Lett. B 323, 65 (1994).

[42] E. Cremmer and B. Julia, Phys. Lett. B 80, 48 (1978).

[43] E. Cremmer and B. Julia, Nucl. Phys. B159, 141 (1979).

[44] S. Weinberg, Phys. Rev. 166, 1568 (1968).

[45] C. G. Callan, Jr., S. R. Coleman, J. Wess, and B. Zumino, Phys. Rev. 177, 2247 (1969).

[46] K. Yamawaki, Phys. Rev. D 35, 412 (1987).

[47] G. Ecker, J. Gasser, H. Leutwyler, A. Pich, and E. de Rafael, Phys. Lett. B 223, 425 (1989).

[48] M. Tanabashi, Phys. Lett. B 384, 218 (1996).

[49] M. C. Birse, Z. Phys. A 355, 231 (1996). 
[50] M. Harada and K. Yamawaki, Phys. Rep. 381, 1 (2003).

[51] K. Kawarabayashi and M. Suzuki, Phys. Rev. Lett. 16, 255 (1966).

[52] Riazuddin and Fayyazuddin, Phys. Rev. 147, 1071 (1966).

[53] E. E. Jenkins, A. V. Manohar, and M. B. Wise, Phys. Rev. Lett. 75, 2272 (1995).

[54] C. G. Boyd and B. Grinstein, Nucl. Phys. B442, 205 (1995).

[55] M.E. Luke and A. V. Manohar, Phys. Lett. B 286, 348 (1992).

[56] A. F. Falk and T. Mehen, Phys. Rev. D 53, 231 (1996).

[57] P. Colangelo, F. De Fazio, and R. Ferrandes, Phys. Lett. B 634, 235 (2006).

[58] J. Brodzicka et al. (Belle Collaboration), Phys. Rev. Lett. 100, 092001 (2008).
[59] B. Aubert et al. (BABAR Collaboration), Phys. Rev. Lett. 97, 222001 (2006).

[60] P. Colangelo, F. De Fazio, S. Nicotri, and M. Rizzi, Phys. Rev. D 77, 014012 (2008).

[61] P. Colangelo, F. De Fazio, and S. Nicotri, Phys. Lett. B 642, 48 (2006).

[62] A. Manohar and H. Georgi, Nucl. Phys. B234, 189 (1984).

[63] J. L. Goity and W. Roberts, Phys. Rev. D 60, 034001 (1999).

[64] M. Di Pierro and E. Eichten, Phys. Rev. D 64, 114004 (2001).

[65] S.-C. Li, T. Wang, Y. Jiang, X. Tan, Q. Li, G.-L. Wang, and C.-H. Chang, Phys. Rev. D 97, 054002 (2018).

[66] G. L. Yu, Z. G. Wang, and Z. Y. Li, Phys. Rev. D 94, 074024 (2016). 